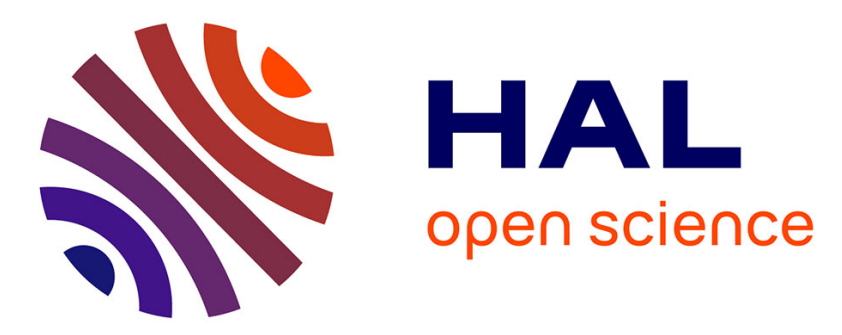

\title{
NMR diffusometry: A new perspective for nanomedicine exploration
}

Florence Franconi, Laurent Lemaire, Jean-Christophe Gimel, Samuel Bonnet, Patrick Saulnier

\section{- To cite this version:}

Florence Franconi, Laurent Lemaire, Jean-Christophe Gimel, Samuel Bonnet, Patrick Saulnier. NMR diffusometry: A new perspective for nanomedicine exploration. Journal of Controlled Release, 2021, 10.1016/j.jconrel.2021.07.025 . hal-03291966

\section{HAL Id: hal-03291966 \\ https://univ-angers.hal.science/hal-03291966}

Submitted on 20 Jul 2021

HAL is a multi-disciplinary open access archive for the deposit and dissemination of scientific research documents, whether they are published or not. The documents may come from teaching and research institutions in France or abroad, or from public or private research centers.
L'archive ouverte pluridisciplinaire HAL, est destinée au dépôt et à la diffusion de documents scientifiques de niveau recherche, publiés ou non, émanant des établissements d'enseignement et de recherche français ou étrangers, des laboratoires publics ou privés. 


\title{
Article type: Review
}

\section{NMR diffusometry: a new perspective for nanomedicine exploration}

Florence Franconi ${ }^{1,2, *}$, Laurent Lemaire ${ }^{1,2}$, Jean-Christophe Gimel $^{1}$, Samuel Bonnet ${ }^{2}$ and Patrick Saulnier ${ }^{1}$

${ }^{1}$ Univ Angers, Inserm, CNRS, MINT, SFR ICAT, F-49000 Angers, France

${ }^{2}$ Univ Angers, PRISM, SFR ICAT, F-49000 Angers, France

* Corresponding author: Florence Franconi

Postal address : MINT/PRISM, Université d'Angers, IBS, CHU d'Angers, 4 rue Larrey, Angers 49933, France

E-mail: florence.franconi@univ-angers.fr

Email and ORCID:

Florence Franconi 0000-0001-6725-0803 florence.franconi@ univ-angers.fr

Laurent Lemaire (0000-0002-1308-9345) laurent.lemaire@ univ-angers.fr

Jean-Christophe Gimel (0000-0001-9865-7390) jean-christophe.gimel@ univ-angers.fr

Samuel Bonnet (0000-0003-0399-1877) samuel.bonnet@ univ-angers.fr

Patrick Saulnier (0000-0002-6408-3533) patrick.saulnier@univ-angers.fr

Declaration of interests: none

DOI: 10.1016/j.jconrel.2021.07.025

Published in Journal of Controlled Release 2021

\begin{abstract}
Nuclear Magnetic Resonance (NMR) based diffusion methods open new perspectives for nanomedicine characterization and their bioenvironment interaction understanding. This review summarizes the theoretical background of diffusion phenomena. Self-diffusion and mutual diffusion coefficient notions are featured. Principles, advantages, drawbacks, and key challenges of NMR diffusometry spectroscopic and imaging methods are presented. This review article also gives an overview of representative applicative works to the nanomedicine field that can contribute to elucidate important issues. Examples of in vitro characterizations such as identification of formulated species, process monitoring, drug release follow-up,
\end{abstract}


nanomedicine interactions with biological barriers are presented as well as possible transpositions for studying in vivo nanomedicine fate.

Keywords Nanomedicine, diffusion, Nuclear Magnetic Resonance, Magnetic Resonance Imaging, Magnetic Resonance Spectroscopy.

\section{Highlights}

- The principles, advantages, drawbacks of NMR diffusometry spectroscopic and imaging methods are presented

- Nanomedicine exploration by NMR diffusometry challenges are discussed

- Representative nanomedicines in vitro and in vivo applications are highlighted

\section{Graphical Abstract}

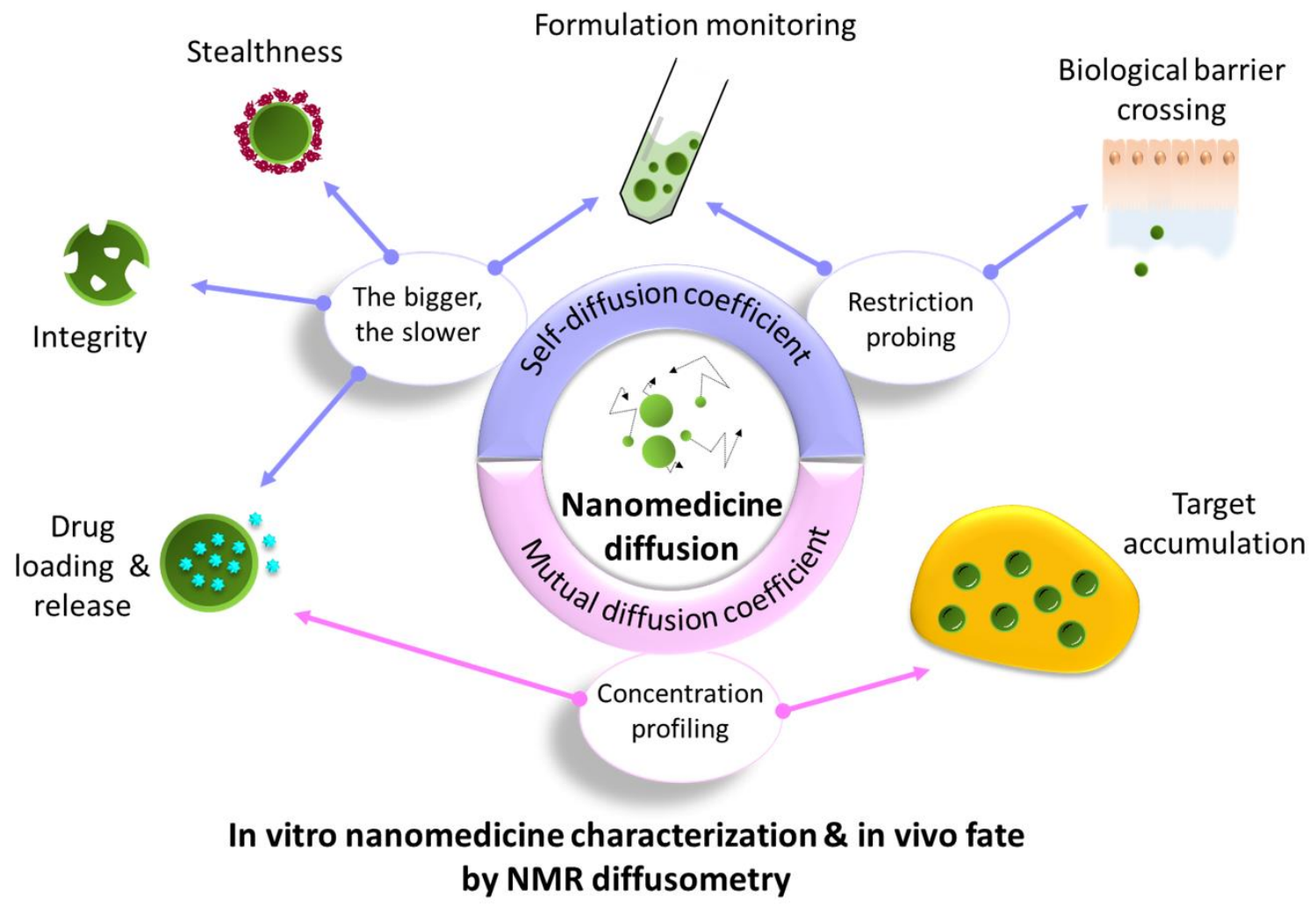




\section{Table of content}

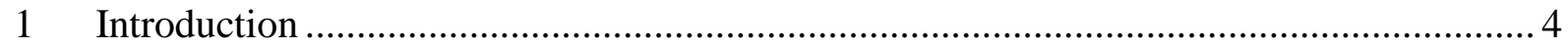

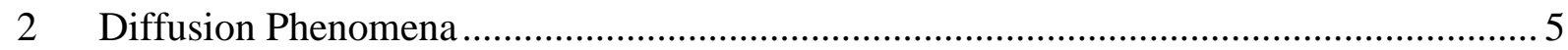

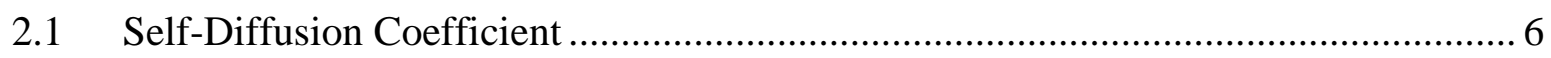

2.2 Mutual-Diffusion Coefficient .........................................................................

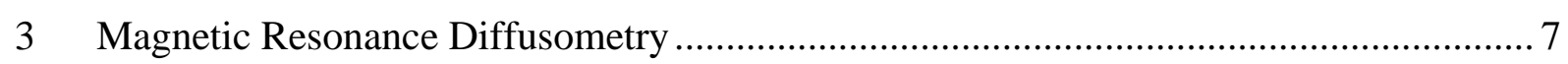

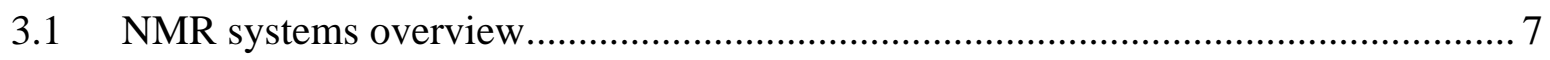

3.2 Self-diffusion coefficients measurement …………......................................... 9

3.3 Mutual-Diffusion Coefficients Measurement...................................................... 11

4 NMR Diffusometry Issues: The Nanoscale Is Not So Simple ………………………..... 11

4.1 Motion Amplitude and Constraint on System Gradient ........................................... 11

4.2 Choice of a Good Marker ……………………............................................. 12

4.3 Quantitative fraction evaluation ................................................................... 12

4.4 Probing medium structure by tuning the diffusion time or by characterizing the non-

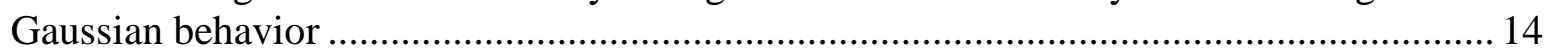

5 Examples of NMR diffusometry contributions to nanomedicines ................................... 14

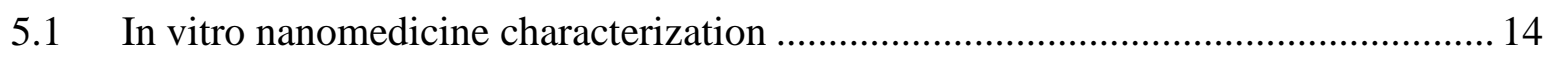

5.1.1 Identification of species in a mixture ……………….................................. 15

5.1.2 Formulation Process Monitoring .................................................................. 15

5.1.3 Interaction Drug-Nanomedicine ............................................................... 16

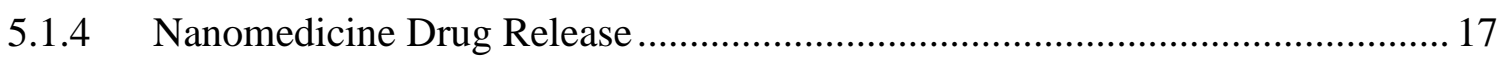

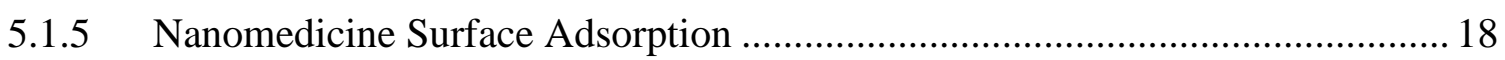

5.1.6 Interaction of Nanomedicine With Biological Barrier ....................................... 19

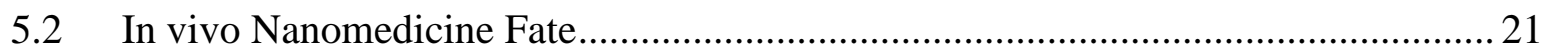

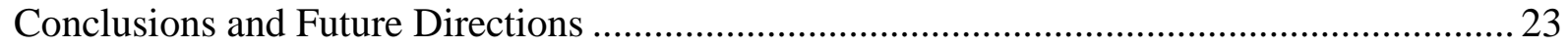




\section{Introduction}

Despite a tremendous amount of research in the last decades, only around fifty nanomedicines were approved by the Food and Drug Administration or the European Medicines Agency and are currently on the market [1]. A legitimate question, therefore, arises about the reasons for such a disappointing outcome [2,3]. Among various factors, the need for a better nanomedicine characterization and a better understanding of their biological behavior were quoted [4].

Indeed, the biological performances of nanomedicines are largely influenced by their physicochemical properties [5]. A precise in vitro characterization is therefore essential to qualify their quality (e.g. size distribution, drug encapsulation), purity (e.g. presence of side products), and process reproducibility. In this context, standardized in vitro characterization procedures have been recently proposed $[6,7]$. Among the panel of available methods [4,6-9], the fast and easy handling dynamic light scattering (DLS) $[10,11]$ is the most popular to evaluate nanomedicine size and distribution. However, this indirect batch method is not optimal for polydisperse systems [11]. Single methods, where nanomedicines are tracked individually, such as Nanoparticle Tracking Analysis (NTA) can be high-resolution alternatives to DLS. However, highly dilute samples are required that can prevent the study of concentration-dependent systems. Characterization of nanomedicines can also be obtained by direct visualization using microscopy-based methods (Scanning, Transmission, or Cryo-Electron microscopy or Atomic Force Microscopy). However, these methods usually require lengthy sample preparations with possible artifacts and do not provide in situ information. More complex in vitro methods are also available such as static scattering techniques [12] that provide structural information or Fluorescence Recovery After Photobleaching to study dynamics of fluorescent nanoparticles in complex bioenvironments [13,14]. Still, there is a real need for a characterization method of native nanomedicine formulation (without sample preparation, labeling, or dilution). Even if not yet widely included in practices, the Nuclear Magnetic Resonance (NMR) diffusometry method is entirely relevant to complete this range of assays and overcome some of the drawbacks $[15,16]$.

There is also a lot to explore to better understand all biological phenomena involved when nanomedicines are administered. Whatever the administration route, nanomedicines will encounter several ambushes on their way to reach their biological target. Intravenously, nanomedicines can be recognized by the immune systems and be eliminated from the circulation [17] via the opsonization with plasma proteins. When other routes are involved (like oral or pulmonary...), nanomedicines must cross epithelium protective layers and cell barriers to reach the bloodstream. Moreover, nanomedicines will have to migrate within the interstitium through the extracellular matrix, especially for subcutaneous or local administrations. The transport through all these complex biological hydrogels is only driven by thermal motions (i.e. the diffusion which is a random motion) and understanding interactions (obstruction, adhesion, lyse...) [18] between nanomedicine and these hydrogels is a crucial point. Finally, therapeutic outcomes will also rely on where and when the carrier will deliver the drug. In all these cases, NMR diffusometry also represents a unique tool. To some point, it is operable in vivo with imaging or spectroscopy abilities, to probe nanomedicine interactions with the bioenvironment.

If improvements in vitro characterization and in vivo nanomedicine fate understanding are essentials, they are also very challenging to address. This is due to the nanometric dimension of objects and the complexity of biological media. We will 
focus this review on the Magnetic Resonance based diffusion spectroscopy and imaging methods which have opened new perspectives in both cases (Fig 1). We believe that the NMR diffusometry, with its new analysis perspectives, can positively contribute to the development of nanomedicines. Even if its potential is still not fully exploited as it often appears as complex, there are no difficult matters to understand, and the highlight of key issues could be essential for readers who are looking for extended characterization tools. After a brief theoretical reminder about diffusion phenomena, principles, advantages, and drawbacks of the method will be highlighted. Then relevant applications of NMR diffusometry to the nanomedicine field will be presented.

\section{Diffusion Phenomena}

Diffusion phenomena are driving the passive transport of species in liquid media. Erratic Brownian motions originate from thermal collisions with solvent molecules surrounding the diffusing species. As far as nanomedicines are concerned, this phenomenon is often the unique force for their displacement in complex biological media and is also referred to as passive transportation.

To describe diffusion phenomena, two different theoretical approaches are used. Both assume random displacements of noninteracting particles but lead to the definition of two different diffusion coefficients as illustrated in figure 2. The self-diffusion coefficient, $\mathrm{D}_{\text {self }}$, is extracted from the knowledge of individual particle trajectories while the mutual diffusion coefficient, $\mathrm{D}_{\text {mut, }}$ is obtained from the temporal evolution of the nanoparticle

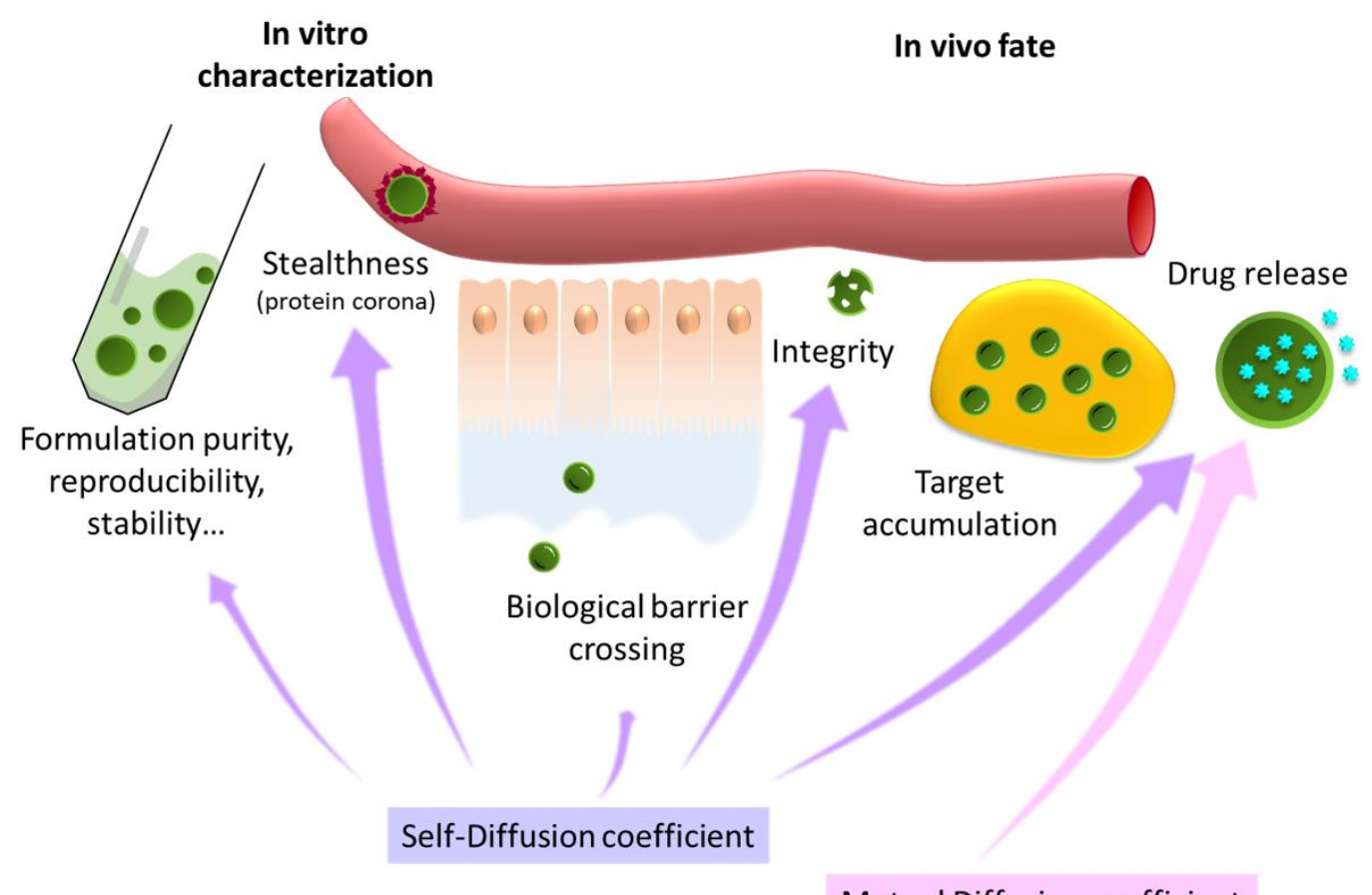

Mutual Diffusion coefficient

Fig. 1 Schematic representation of in vitro and in vivo nanomedicine characterization issues and the potential contribution of NMR diffusometry to the nanomedicine field. 


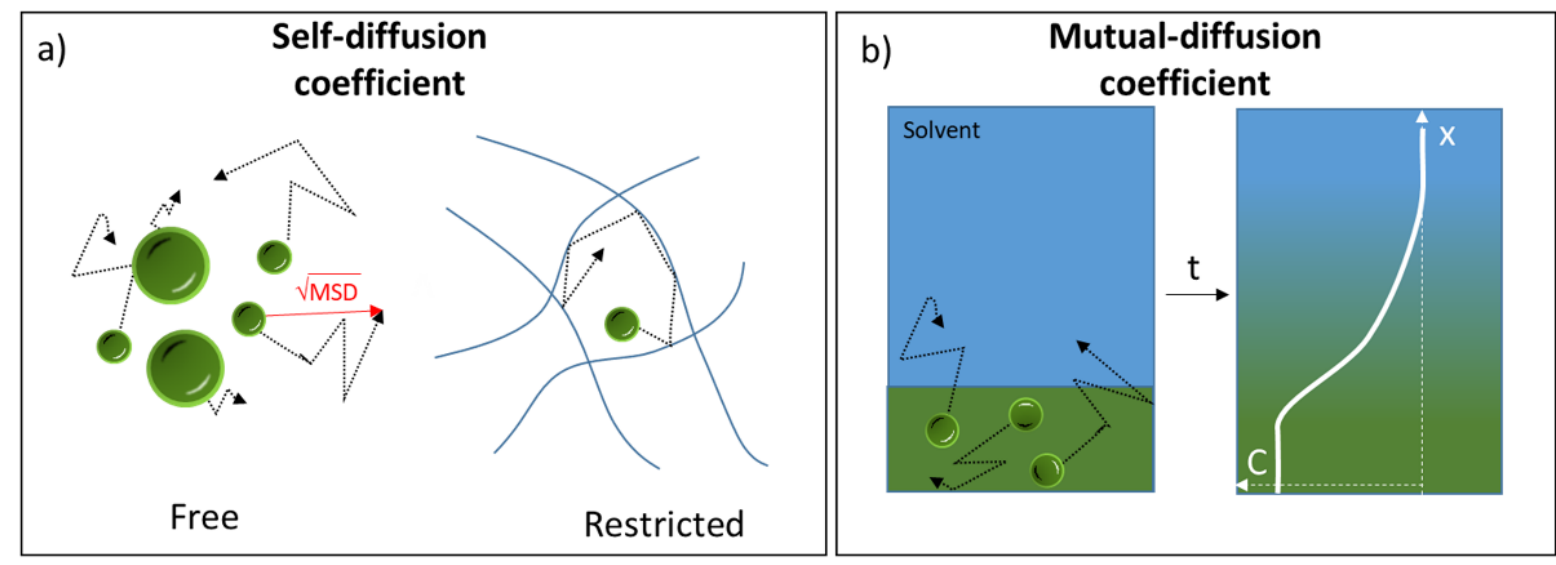

Fig. 2 Schematic representation of the self-diffusion and mutual diffusion coefficients. $\boldsymbol{a}$ The self-diffusion coefficient is derived from individual particle trajectories using the mean square displacement MSD. When species diffuse inside an obstructed, confined, or porous medium, the self-diffusion is called restricted in opposition to the free diffusion observed when no geometrical restrictions are present. $\boldsymbol{b}$ The mutual diffusion coefficient characterizes the dynamic of net mass transport of one species in the presence of concentration gradients.

concentration profile. Both are expressed in $\mathrm{m}^{2} / \mathrm{s}$ but relate very different physical quantities: the mean square displacement (MSD) to the time for $\mathrm{D}_{\text {self }}$ (see equation 2) and the flux of particles to the concentration gradient for $D_{\text {mut }}$ [19] (known as first Fick's law). For highly diluted suspensions (below $\sim 0.5 \%$ volume fraction), $\mathrm{D}_{\text {self }}$ and $\mathrm{D}_{\text {mut }}$ are equal and are referred to as $\mathrm{D}_{0}[20,21]$. However, in practice for NMR experiments, measurements are performed at intermediate or even high concentrations to ensure a sufficient signal-to-noise ratio. In this case, $D_{\text {self }}$ and $D_{\text {mut }}$ have very different behavior even if they both tend to zero at very high concentrations due to steric hindrance that freeze particle motions (Fig. 3).

$\mathrm{D}_{0}$ is related to the friction felt by the nanomedicine in the medium. In general, the bigger the nanomedicine, the slower the diffusion. And for a very diluted suspension of spheres in an isotropic and continuous medium of viscosity $\eta$, the Stokes-Einstein equation states that the diffusion is related to the hydrodynamic radius $\mathrm{R}_{\mathrm{h}}$ via equation 1, with $\mathrm{k}$ the Boltzmann's constant and $\mathrm{T}$ the absolute temperature.

$$
\begin{aligned}
& D_{0}=\frac{k \cdot T}{6 . \pi \cdot \eta \cdot R_{h}} \\
& (\text { Equation } 1)
\end{aligned}
$$

\subsection{Self-Diffusion Coefficient}

The self-diffusion coefficient $\mathrm{D}_{\text {self }}$ characterizes the distance an individual species such as a particle would likely diffuse in a fixed interval of time. The selfdiffusion coefficient relates the mean square displacements (MSD) observed during the diffusion observation time $t$ using the Langevin approach [19]:

$M S D=6 . D_{\text {self }} . t$

dimensional

(Equation 2)

In the case of NMR studies, the selfdiffusion coefficient estimation is averaged over a population of objects and is usually performed at the thermodynamic equilibrium. The diffusion observation time corresponds in this case to $\Delta$, the delay between the two diffusion sensitizing 
magnetic field gradients of the NMR sequence (figure 5a).

The measurement of the self-diffusion coefficient can provide valuable information on nanomedicine formulations: probe the species in presence by measuring their self-diffusion coefficient and infer the size of objects with precaution when concentration effects cannot be neglected.

When the species move inside an obstructed, confined, or porous medium such as a hydrogel, their diffusion can be hindered, and $\mathrm{D}_{\text {self }}<\mathrm{D}_{0}$ will then be defined as restricted or apparent in opposition to the free diffusion (Fig. 2 and 3) observed when no geometrical restrictions are presents. The restricted diffusion coefficient will depend on the topology of the medium (porosity, tortuosity...), the characteristics of the diffusing species (size, shape ...), and possible interactions between the medium and the species. The time scale of diffusion is also important as the duration of the observation time will determine the displacement of the particles observed. If the observation time is long enough, the particles will diffuse far enough to experience the effect of restriction and have their transports limited by the boundary encountered. In this case, the restriction of diffusion can provide non-invasively information about the sub-micron structure of the medium network and about how a particle can diffuse inside this medium. In the nanomedicine domain, this way to probe the environment structure [22] has shown its utility to study the interaction between nanomedicines and the biological barriers they will have to cross (Fig. 1).

\subsection{Mutual-Diffusion Coefficient}

The mutual diffusion coefficient is related to the net collective motion of the diffusing species in the presence of a concentration gradient and therefore is also called collective or cooperative diffusion coefficient in the literature $[23,24]$. Induced mass flux is observed and can be described by Fick's laws [19]. However, it must be kept in mind that the diffusing objects do not feel any concentration gradients that could determine the direction of individual motions, they simply undergo random displacements but the resultant species spreading appears as a move from high to low concentration domains.

Mutual diffusion coefficients can be determined by NMR from the concentration (C) profiles measured during the release or uptake through an interface. In one dimension, the diffusion equation can be written:

$\frac{\delta C}{\delta t}=D_{m u t} \cdot \frac{\delta^{2} \cdot C}{\delta x^{2}}$

(Equation 3)

with $\mathrm{t}$ being the time and $\mathrm{x}$ the position (length). In general, the diffusion equation is difficult to handle, and mathematical solutions only exist for specific initial concentration profile $\mathrm{C}(\mathrm{t}=0)$ and boundary conditions [25].

For nanomedicine, mutual diffusion coefficient could then be particularly helpful to study drug release or interface crossing (Fig. 1).

\section{Magnetic Diffusometry \\ Resonance}

\subsection{NMR systems overview}

Nuclear Magnetic Resonance (NMR) techniques cover a range of noninvasive analysis methods, all based on the signal produced by magnetic nuclei, mainly ${ }^{1} \mathrm{H}$, polarized by a strong magnetic field, and exposed to radiofrequency excitation. A wide range of systems exist from NMR spectrometers to magnetic resonance imaging (MRI) scanners and including Time-Domain NMR (TD-NMR) systems (Figure 4). Magnetic field intensity (for sensitivity) and magnetic field gradients intensity (for diffusion weighting) are determinant system characteristics for accurate diffusion coefficient estimation. 


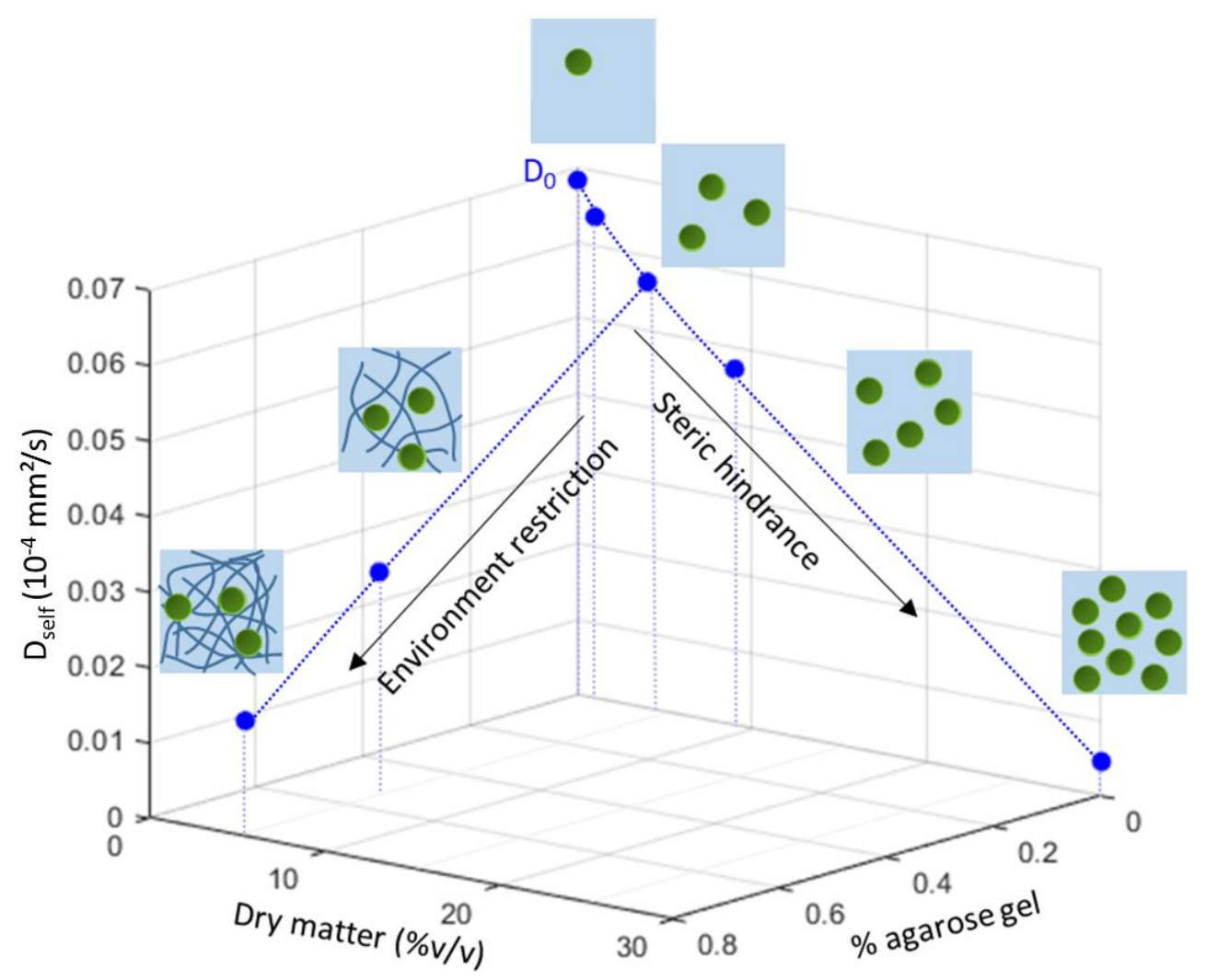

Fig. 3 Self-diffusion coefficients measured using PFG-NMR method of a $60 \mathrm{~nm}$ diameter lipid nanocapsules suspension in $\mathrm{D}_{2} \mathrm{O}$ at $25^{\circ} \mathrm{C}$. Evolution according to nanocapsules dry matter percentage to study steric hindrance influence and according to agarose gel percentage in the medium to study environment restriction. Diffusion experiments in agarose were performed at 5\% lipid nanocapsules dry matter [unpublished results from JC Gimel, TAT Do, and F Franconi].

The NMR spectrometer with usually a vertical magnet producing intense magnetic field (up to $23.5 \mathrm{~T}$ corresponding to ${ }^{1} \mathrm{H}$ frequencies in the $1 \mathrm{GHz}$ range) and a small bore are known to offer sensitivity and spectral resolution on a whole sample of limited volume (usually $5 \mathrm{~mm}$ diameter tube, with $\sim 500 \mu \mathrm{L}$ samples). To perform diffusion measurement, NMR spectrometers need to be equipped with magnetic field gradients capabilities.

MRI scanners, with most of the time horizontal magnet, offer a large range of magnetic field (generally from $3 \mathrm{~T}$ to $11.7 \mathrm{~T}$ for preclinical applications and from $1.5 \mathrm{~T}$ to $7 \mathrm{~T}$ for clinical application, even if $21 \mathrm{~T}$ preclinical systems exist and $14 \mathrm{~T}$ human systems are planned). Their larger bore size (several tens of centimeters for the clinical systems) allows in vivo scanning on human or animal. They possess by default magnetic field gradients to localize the signal to produce images which can also be used for diffusion measurements. Not only images can be acquired on imaging systems but also spectra, either on a voxel by localized magnetic resonance spectroscopy (MRS) or on a matrix of voxels by chemical shift imaging (CSI). NMR and MRI systems are usually accessible in core facilities (hospital, university, private sector...) to mutualize expenses and expertise. 


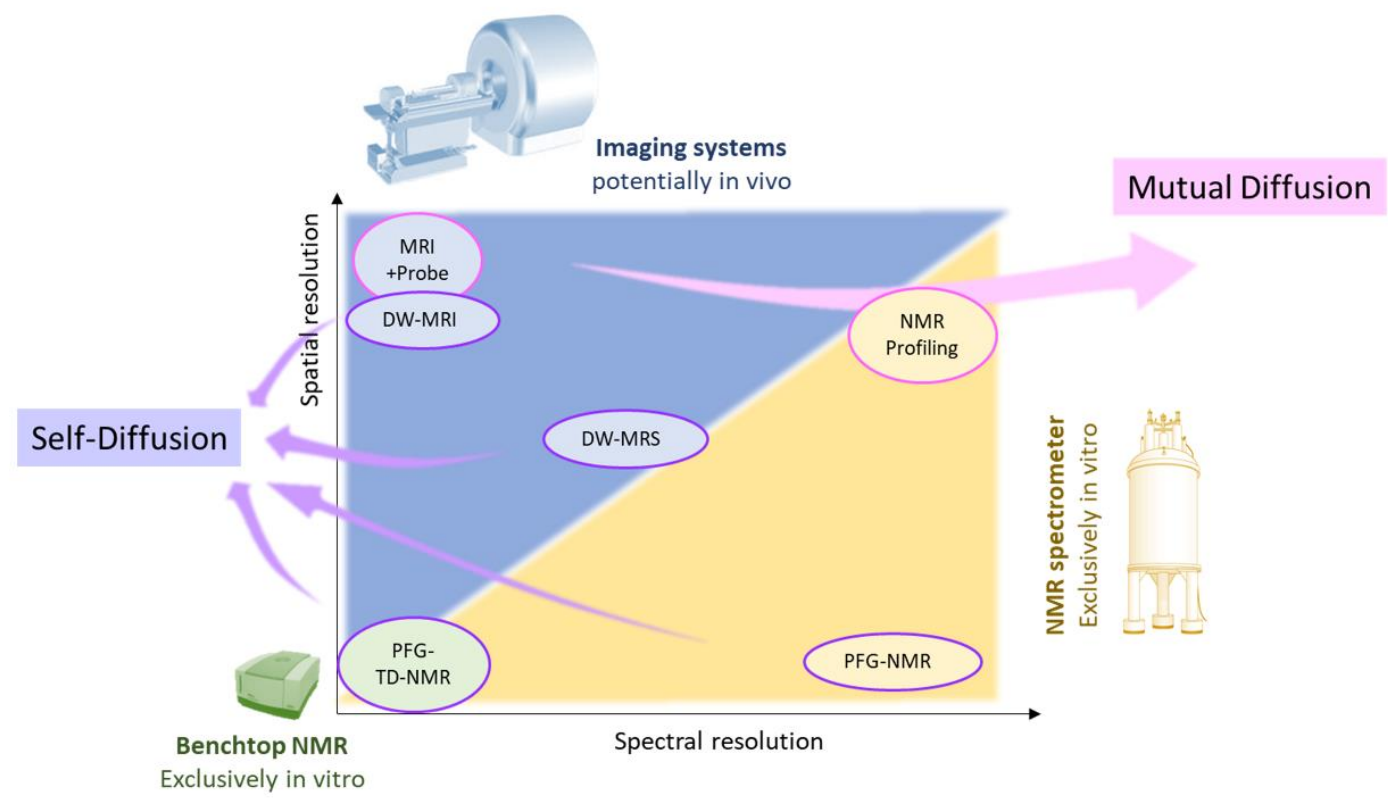

Fig. 4 Overview of NMR systems and corresponding NMR diffusometry methods to perform self and mutual diffusion coefficients estimation. For self-diffusion measurement, diffusion weighting $(D W)$ is obtained by the presence of two diffusion sensitizing magnetic field gradient pulses coupled either with imaging (MRI) or localized spectroscopy (MRS) on imaging systems. On NMR spectrometers, a similar diffusion pattern for NMR spectroscopic acquisition is applied and called PFG for pulse field gradient. For mutual diffusion coefficient estimation, nanomedicine concentration profile evolution during release or uptake is measured. The nanomedicine NMR visibility is obtained either by labeling with a magnetic probe or by using a specific resonance frequency signal.

Bench top cheaper low magnetic field systems, accessible for individual laboratories, also exists (from $0.2 \mathrm{~T}$ up to $2 \mathrm{~T}$ ) to measure diffusion coefficients on an entire sample of limited volume but without any spectral or spatial resolutions.

\subsection{Self-diffusion measurement}

The measurement of self-diffusion with NMR systems is based on Stejskal and Tanner's work [26]. The idea relies on the weighting of the NMR signal by the diffusion-induced translational motions in the presence of two diffusion-sensitizing magnetic field gradient pulses. Signal attenuation is then observed, it dependents on both the diffusion coefficient and on the degree of sensitization to the diffusion of the pulse sequence represented by the diffusion weighting factor $b$ :

$b=\gamma^{2} \cdot G^{2} \cdot \delta^{2} \cdot\left(\Delta-\frac{\delta}{3}\right)$

(Equation 4)

where $\gamma$ is the gyromagnetic ratio and $\delta, G$ and $\Delta$ are respectively the gradient duration, intensity, and separation (Fig. 5a). $\Delta$ Also corresponds to the diffusion observation time during which the diffusion is probed. $D_{\text {Self }}$ can then be estimated from the exponential decay of the NMR signal induced by incremental diffusion weighting according to:

$S(b)=S_{0} \cdot e^{\left(-b \cdot D_{\text {self }}\right)}$

(Equation 5) 

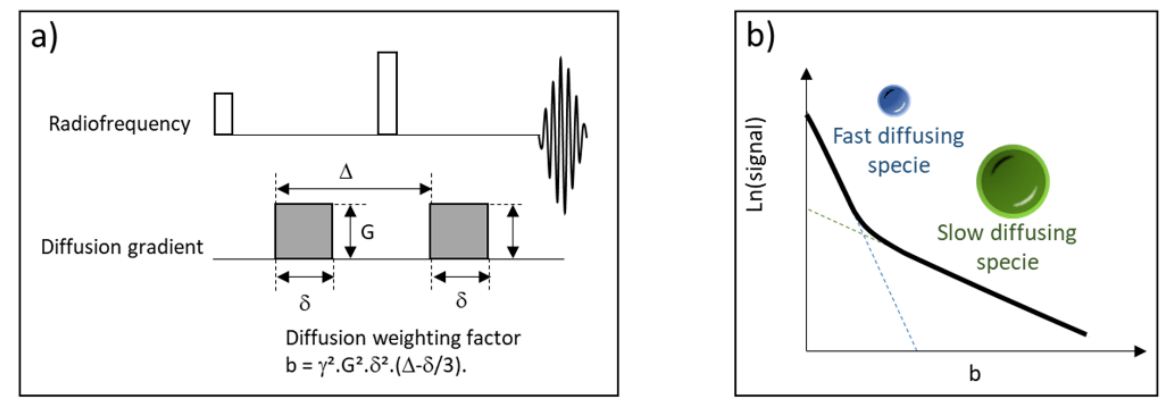

I. Laplace inverse transform
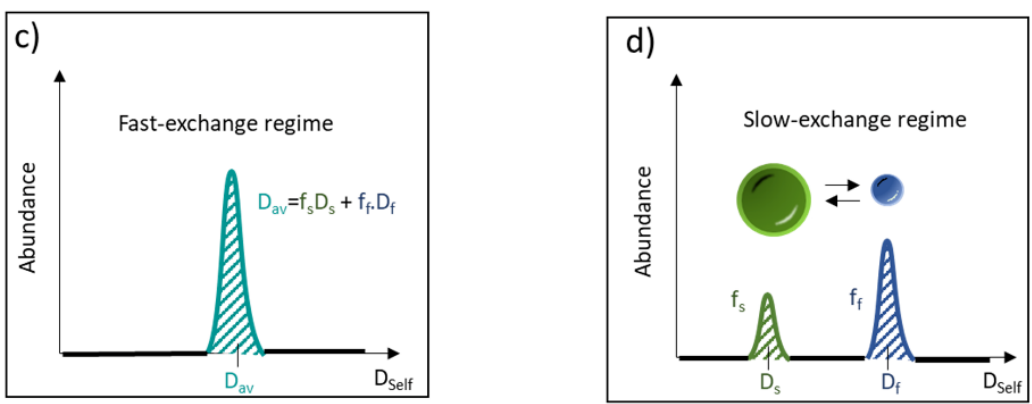

Fig. 5 a Self-diffusion coefficient estimation is based on the acquisition of a signal weighted by diffusion by two diffusion magnetic field gradient pulses. Signal attenuation is observed depending on the diffusion weighting factor $b . b$ is equal to $\gamma^{2} \cdot G^{2} . \delta^{2} .(\Delta-\delta / 3)$ with $\gamma$ the gyromagnetic ratio and $\delta$, $G$ and $\Delta$ respectively the diffusion gradient duration, intensity, and separation. $\boldsymbol{b}$ Diffusion coefficients are estimated from the exponential decay of the NMR signal induced by incremental diffusion weighting. $\boldsymbol{c}$ When a molecule is in fast exchange during the diffusion observation time $\Delta$ between two states, for example, free and bound drug to a nanomedicine, a single diffusion coefficient will be observed which will be a population average of the coefficients of each population. $d$ With no or slow exchange, two distinct diffusion coefficients will directly be measured with an intensity relative to each population.

where $S(b)$ and $S_{o}$ are respectively the signal amplitude in the presence and absence of diffusion gradients (Fig. 5b).

On NMR spectrometers, self-diffusion coefficients are estimated from pulsed-field gradient (PFG) based sequences using either spin or stimulated echo patterns. This method is sometimes named DOSY (Diffusion Ordered SpectroscopY) due to the processing tool associated. Species contributing to the signal can be separated based on both the spectral and the diffusion dimensions.
The same PFG method is also used by a benchtop time-domain NMR systems without spectral separation. This method has been widely used for droplet size measurements in either oil-in-water or water-in-oil food emulsions. However, even if droplet size as low as $250 \mathrm{~nm}$ could potentially be analyzed by TD-NMR systems, we did not find any report of an application in the nanomedicine field.

A similar diffusion sensitizing pattern can also be applied to imaging to obtain diffusion-weighted imaging (DW-MRI). 
Water signal DW-MRI is routinely performed in the clinical context. If the diffusion gradient implementation in conventional spin-echo-based sequences has been proposed long ago, it's the implementation in echo-planar sequence [27] that has largely contributed to its development in clinical practices. Indeed, the possibility to acquire multiple diffusion directions rapidly with a low level of motion artifacts was determinant to obtain good quality diffusion tensor imaging to perform fibers tractography. The main advantage of imaging resides in the potential to probe diffusion spatial heterogeneities, especially in vivo. Midway between PFG-NMR and DW-MRI, imaging systems can measure diffusion coefficients with spectral separation but on a single localized volume using diffusion-weighted magnetic resonance spectroscopy (DW-MRS).

\subsection{Mutual-Diffusion Measurement}

Coefficients

To measure the mutual-diffusion coefficient, spatial resolution is mandatory to evaluate the nanomedicine concentration profile evolution during species release or uptake. If the spatial resolution is evident for imaging systems and allows to study of the diffusion even in 3 dimensions, mutualdiffusion can also be performed with NMR spectrometers using NMR profiling but only in one dimension. However, nanomedicines need to be NMR visible or labeled to be distinguished from the surrounding medium. Most of the time, a contrast agent is required, using either labeled nanomedicine companion [28] or co-drug/label loading of the nanomedicine [29]. Most of the contrast agents useable in MRI are not seen directly on the image. They shorten the relaxation times of surrounding water molecules, proportionally to their concentration. Paramagnetic centers, such as gadolinium or manganese ion, or superparamagnetic centers, such as iron oxide particles can be used to respectively induce positive or negative image contrast. However, it could be sometimes tricky to convert signal profile into concentration profile and calibration steps or relaxation time quantification could be valuable. Another way to track nanomedicines is to acquire a specific resonance frequency signal associated with the nanomedicine, different from the water one, such as fat for ${ }^{1} \mathrm{H}$ [30] or fluorine-19 [31] for heteronuclei. In this case, the signal is directly proportional to the concentration of nuclei but lower sensitivity or reduced spatial resolution is often observed compared to labeling strategies.

It must be pointed out that self-diffusion and mutual diffusion NMR measurement techniques are not in the same length scales nor time ranges. Travel distances are in the range of micrometers during milliseconds to second for $\mathrm{D}_{\text {self }}$ estimation while travel distances are in the millimeters range with observation times from minutes to hours for $\mathrm{D}_{\text {mut }}$ estimation.

\section{NMR Diffusometry Issues: The Nanoscale Is Not So Simple}

If most of the advantages of nanomedicines are linked to their small size, this also represents a real challenge when characterization and analysis are required. The application of NMR diffusometry to the nanomedicine field is not straightforward and several issues must be addressed.

\subsection{Motion Amplitude and Constraint on System Gradient}

To measure self-diffusion, the range and sampling scheme of the diffusion weighting factor $b$ impacts the accuracy of diffusion coefficient estimate [32]. The expected diffusion coefficients of a large object such as nanomedicine are small and therefore require large maximum $b$ values. The high gradient strength capacity of the NMR system is therefore essential. In imaging systems, magnetic field gradients are usually quite low, around $0.05 \mathrm{~T} / \mathrm{m}$ in clinical systems and between $0.2 \mathrm{~T} / \mathrm{m}$ to 
$1 \mathrm{~T} / \mathrm{m}$ in preclinical systems. Gradient Intensity can reach $4 \mathrm{~T} / \mathrm{m}$ in benchtop TDNMR systems while in NMR spectrometers, dedicated probes offer a wide range of magnetic field gradients from $0.5 \mathrm{~T} / \mathrm{m}$ up to $30 \mathrm{~T} / \mathrm{m}$. As an example, the diffusion coefficient of a $100 \mathrm{~nm}$ diameter nanomedicine in water at $25^{\circ} \mathrm{C}$ is low, $\sim 5$ $\mu \mathrm{m}^{2} / \mathrm{s}$, compared to the water's $(2300$ $\mu \mathrm{m}^{2} / \mathrm{s}$ ) [33]. To correctly estimate them, large maximum $b$ values are then required. An optimal maximum $b$ value $\sim 5 \mathrm{~s} / \mathrm{m}^{2}$ can be predicted from variance minimization [32] for a linear b sampling pattern. This maximum $b$ value can be reached using $a$ diffusion time of $\Delta=50 \mathrm{~ms}$ for a gradient system maximum intensity of $0.5 \mathrm{~T} / \mathrm{m}$ at the cost of a disadvantageous high gradient diffusion duration $(\Delta=27 \mathrm{~ms})$ while with a stronger gradient system, capable of $5 \mathrm{~T}$, the gradient duration can be lower down to $2.5 \mathrm{~ms}$, impacting proportionally the sequence echo time. Large objects are also characterized by reduced tumbling rate causing unfavorable fast transverse spin relaxation (T2). When long echo time is required, large signal attenuation is observed. Therefore, when nanomedicines are involved, high magnetic field gradient strength are particularly of interest to correctly sample the diffusion induced decay and to limit the echo time length responsible for a poor signal-to-noise ratio by high relaxation weighting. In brief, the gradient characteristics of the NMR system will determine the range of diffusion coefficients that can be measured.

\subsection{Choice of a Good Marker}

One of the advantages of the self-diffusion measurement based on the spectroscopic method (PFG-NMR, DW-MRS) is the ability to simultaneously monitor NMR signals at different chemical shifts to separate diffusing components. Each signal can shed a different light. If the signal corresponds to a molecule part of the nanomedicine components, the selfdiffusion coefficient could be a good marker of the nanomedicine assembly state or the modification of the nanomedicine mobility induced by interaction or obstruction of the surrounding environment. If the signal originates from a coating molecule, the self-diffusion coefficient can reveal integrity losses. If the signal is issued from a drug carried by the nanomedicine, the self-diffusion coefficient could be a good marker of the interaction strength between the drug and the nanomedicine or of the ratio of drug encapsulated or released. If the signal comes from the surrounding medium in which the nanomedicines diffuse, the selfdiffusion coefficient will shed light on the possible interactions between the nanomedicine and the surrounding medium such as the protein from the biological fluid which can adsorb on the nanomedicine surface or the mucus modifications upon mucolytics nanomedicine action. All these issues will be illustrated in the following parts. However, whatever the signal origin, they are generally low intensity compared to the large water signal. Though, usually, the water signal does not represent a problem as it can be suppressed by presaturation or by taking advantage of the difference of diffusion coefficients which make the water signal much rapidly attenuated than nanomedicine one even for relatively small $b$ values. If not, the solvent can be replaced in vitro with a deuterated analog with potentially higher costs, less biological compatibility, and possible interaction on the formulation itself.

\subsection{Quantitative fraction evaluation}

If the estimation of the self-diffusion coefficient can elucidate the species present in the formulation or inform on the surrounding media structure, it could also be important to quantify the fraction of ${ }^{1} \mathrm{H}$ nuclei involved in each diffusing species to quantify the degree of nanomedicine assembly or the fraction of drug released.

In the case of dynamic exchange of the analyzed marker molecule between two compartments (for example, free and bound 
for drugs or monomer and micelle for surfactant) during the diffusion observation time, two distinct diffusion coefficients are measured in the slow exchange rate regime, with relative intensity for each population. The contribution determination of each compartment (slow or fast diffusing components) to the multiexponential signal decay with increasing diffusion weighting factor is a tricky task, mainly based on the non-trivial inverse Laplace transform (Fig. $5 \mathrm{c}$ and d) to obtain the continuous distribution of diffusion coefficients. In this case, the signal decay expressed in equation 5 should be replaced by the following integral equation:

$S(b)=\int_{0}^{\infty} h(D) \cdot e^{(-b \cdot D)} \cdot d D$

(Equation 6)

with $h(D)$ the distribution function. This is an ill-posed mathematical problem, especially when noise is present and/or when the attenuation is not completely sampled, meaning that an infinite number of solutions are consistent with the experimental dataset. Regulators are therefore applied to make the inversion meaningful as proposed by different processing methods. Methods that process each peak independently such as CONTIN [34] are called univariate while multivariate approaches such as DECRA [35] or SCORE [36] analyze simultaneously the whole dataset. The free GNAT toolbox [37] regroups a range of convenient PFG-NMR processing methods. Whatever the method, acquired dataset quality is primordial and an accurate determination of the diffusion coefficient distribution remains a delicate step.

When the exchange rate between both populations is fast on the NMR observation times scale, a single diffusion coefficient $\mathrm{D}_{\text {self }}$ is observed which is a populationweighted average between both diffusion coefficients according to:

$D_{\text {self }}=f_{\text {free }} \cdot D_{\text {free }}+f_{\text {bound }} \cdot D_{\text {bound }}$ (Equation 7) where $D_{\text {free }}$ and $f_{\text {free }}$ are the diffusion coefficient and the fraction of the free species respectively, while $\mathrm{D}_{\text {bound }}$ and $\mathrm{f}_{\text {bound }}$ are the diffusion coefficient and the fraction of the bound or assembled system respectively. The free diffusion coefficient is often quite easy to evaluate, using a dilute solution for self-assembled systems (below critical aggregation concentration) or a nanoparticle-free solution for the drug. The bound diffusion coefficient is often more delicate to estimate. For example, the drug bound to polymeric nanoparticle diffusion coefficient can be estimated by assuming its equality to the polymer signal diffusion coefficient $[38,39]$. However, this fraction quantification is most of the time not absolute but only reflects the NMR visibility of each population. Indeed, the echo-based PFG-NMR peak intensities are relaxation weighted, so, signal attenuations depend on the related population relaxation characteristics. Therefore, the high diffusion observation time $\Delta$ and the high gradient duration $\delta$ required for analyzing the slow diffusing nanomedicine act differently on the NMR visibility. The gradient duration $\delta$ influences the sequence echo time and therefore the $\mathrm{T} 2$ relaxation time weighting of the observed signal. The diffusion observation time $\Delta$ also acts on the relaxation signal weighting but differently depending on the sequence applied. In spinecho-based sequences, the diffusion observation time $\Delta$ acts on the echo time and consequently on the transverse $\mathrm{T} 2$ relaxation time weighting while in stimulated echo-based sequence, the diffusion observation time $\Delta$ acts on the mixing time and consequently on the longitudinal $\mathrm{T} 1$ relaxation time weighting. In brief, nuclei part of a large object such as nanomedicine often has shorter transversal relaxation times that lead to their underestimation compared to free molecules. However, more complex methods exist to take into account relaxation effects and to reach absolute quantitative fraction estimation [40,41]. 


\subsection{Probing medium structure by tuning the diffusion time or by characterizing the non-Gaussian behavior}

The structure of the surrounding medium can be probed by measuring the species' self-diffusion coefficient. The diffusion observation time $\Delta$ determines the length of the possible displacement of the object. As an example, the root-mean-squaredisplacement of a $100 \mathrm{~nm}$ diameter particle during a diffusion observation time $\Delta$ of $20 \mathrm{~ms}$ is $\sim 0.8 \mu \mathrm{m}$ while during a diffusion time of $200 \mathrm{~ms}$ it reaches $\sim 2.5 \mu \mathrm{m}$. For very short diffusion times, particles are less sensitive to any constraint and move quasi freely. Their $\mathrm{D}_{\text {self }}$ is very close to $\mathrm{D}_{0}$, the one in absence of obstruction (i.e. in pure solvent). When the observation diffusion time increases, diffusing species start feeling the restriction from the surrounding medium, and the measured $D_{\text {self }}$ decreases as $\Delta$ increases. At very large $\Delta, D_{\text {self }}$ reaches a plateau which is the macroscopic diffusion coefficient [42-45]. The observed diffusion coefficient reflects the confined geometry characteristics of the medium (interstices, droplets, pores, obstruction, tortuosity...). An obstruction factor [38] can be estimated according to $\mathrm{D}_{\text {Self }} / \mathrm{D}_{0}$. Modeling has also been proposed to derive the network structure from diffusion measurements [42,46-48].

Another way to characterize the impact of compartments and barriers is to estimate how the probability distribution of a particular species displacement in a given time duration deviates from a Gaussian distribution, which is the expected behavior in a simple liquid. A Kurtosis parameter, K, can then be estimated which quantifies the non-Gaussian degree of the probability distribution. This parameter can help to evaluate the degree of diffusion coefficient variability inside a voxel [49,50]. Diffusivity is then analyzed using a polynomial model:

$$
\begin{aligned}
& S(b)=S_{0} \cdot e^{\left(-b \cdot D_{\text {self }}+b^{2} \cdot D_{\text {self }}{ }^{2} \cdot \frac{K}{6}\right)} \\
& \text { (Equation 8) }
\end{aligned}
$$

The kurtosis effect is mainly observed for high $b$ values as a deviation from the monoexponential model, resulting in a reduced apparent diffusion coefficient. To our knowledge, only water diffusion kurtosis magnetic resonance imaging has been performed until now to probe heterogeneous and irregularity of in vivo microstructures but none of the works was in the nanomedicine field even if nonGaussian diffusion of tracers in mucin has yet been demonstrated, especially at low $\mathrm{pH}$ where the hydrogel heterogeneity is higher [51].

\section{Examples of NMR diffusometry contributions to nanomedicines}

\subsection{In vitro nanomedicine characterization}

As already quoted, the physicochemical characterization (size, shape, surface, polydispersity, aggregation...) of nanomedicine formulation is mandatory to understand their biological behavior. The reproducibility and stability of formulation are also crucial for the ability to scale up nanomedicine formulation. However, even if a panel of assays exists, the need for new methods is still relevant to overcome characterization challenges. This characterization can largely benefit from the in vitro NMR diffusometry analysis, reinforced by the chemical selection offered by the spectral separation of spectroscopy. Therefore, most of the formulation characterization studies presented in this section were obtained with NMR spectrometers. 


\subsubsection{Identification of species in a mixture}

The identification of species presents in a nanoformulation is most of the time based on size determination (Fig. 6a). Selfdiffusion coefficients estimated by PFGNMR are converted into hydrodynamic radii using the Stokes-Einstein relation (Equation 1). The ability of PFG-NMR to determine size has been demonstrated for micelles [38,52] or reverse micelles [53] using the surfactant NMR signal but also for gold nanoparticles using the protective groups NMR signal [54].

It is important to highlight the importance of orthogonal characterization in the nanomedicine field, each method being complementary to the others. The standard methods can present some drawbacks that can be problematic for nanomedicine characterization. For example, the sample preparation for transmission electron microscopy could sometimes alter the sample and impact the size measurement or the DLS method presents a distorted sensitivity to large components as scattered light intensity is proportional to particles diameter to the 6th power. In contrast, PFGNMR diffusometry presents several advantages. It is noninvasive, neither complex sample preparation nor exogenous probes are required and chemical specificity is offered by the spectral separation. Furthermore, the method is free from potential interference of large particles therefore, contrary to DLS, small objects, even if they are mixed with a larger one, can be picked out. Indeed, the NMR signal is proportional to the number of ${ }^{1} \mathrm{H}$ nuclei involved weighted by the relaxation effects (smaller species with longer relaxation times yielding to sharper NMR peaks than larger ones) which favor the visibility of a small object. Guyon et al. [55] have shown that PFG-NMR was able to identify the main nanoassembly population that was masked in DLS by small traces of large aggregates. Whereas, small formulation residual components such as micelles which can be masked in DLS by the larger main nanomedicine population could be detected [56,57] using PFG-NMR. PFG-NMR is also particularly powerful to provide polydispersity profiles of a complex mixture such as what was done on the commercial polymer-based nanopharmaceuticals Copaxone $®[58]$. The efficiency of purification methods to eliminate residual micelles or stability issues by assessing the absence of modification upon storage could also take benefits using diffusometry techniques.

\subsubsection{Formulation Process Monitoring}

The diffusion measured by PFG-NMR can provide valuable information to monitor the formulation process (Fig. 6b). For example, the degree of nanomedicine self-assembly can be quantified from the measurement of the fractions of molecules free and assembled or bound to assembly. The ability to monitor a micellization process $[38,59]$ using the surfactant NMR signal diffusion has been demonstrated as well as the promoting role of lysine in the stabilization of self-assembly of inorganic materials to form silica nanoparticles using the lysine NMR signal [60]. Nanoemulsion formulation can also be studied by measuring the diffusion coefficients evolution with the diffusion observation time $\Delta$ to explore the restricted character of the colloidal phase. Continuous phases generally have unrestricted diffusion coefficient while dispersed phase have restricted one. D'agostino et al. [61] have assessed the structure evolution during nano-emulsification by estimating the diffusion coefficients of water, oil, and surfactant signals as a function of the water percentage. In the absence of water, a biphasic oily/surfactant unrestricted diffusion was observed despite droplet presence but with a size too large on the NMR observation time scale to limit the displacement. At the inversion phase, a bicontinuous water and oil structure was observed. Above the inversion phase, water was still a continuous phase with 
unrestricted diffusion while the oily phase became dispersed with its diffusion coefficient showing dependence on the observation time scale.

\subsubsection{Interaction Drug-Nanomedicine}

The mechanisms of drug interaction with the carrier (encapsulated, entrapped, dissolved, covalently bond, adsorbed on the surface) are crucial to understand drug release phenomena. PFG-NMR diffusometry can be applied to help to elucidate mechanisms of drug inclusion and location (Fig. 6c). The estimation of the drug diffusion coefficient can shed light on drug carrier bonding while a polymer one, as long as it is constitutive of the nanomedicine, can be used to evaluate the drug bound diffusion coefficient, assuming they have the same diffusional properties.
When the drug interacts with the carrier, a reduction of diffusion coefficients is generally observed related to the size modification or the strength of interaction. For example, supramolecular complex formation of a cytostatic drug, gemcitabine, with its cucurbuturil nanocontainer was proved by PFG-NMR by the reduction of gemcitabine diffusion coefficient when complexes as well as the possible impact of ethanol consumption[62,63]. Similarly, the covalent attachment of an antiinflammatory and anti-cancer drug, the histone deacetylase inhibitor valproic acid, to its polymeric nanocontainer was demonstrated with PFG-NMR[64]. The stronger the association between the drug and the carrier, the larger is the decrease of the diffusion coefficient. This was demonstrated by Ivanova et al. [65] for antibiotic drugs (daunorubicin, DAU) loaded polymeric nanoparticles. A fraction of free to bound drug may even be estimated
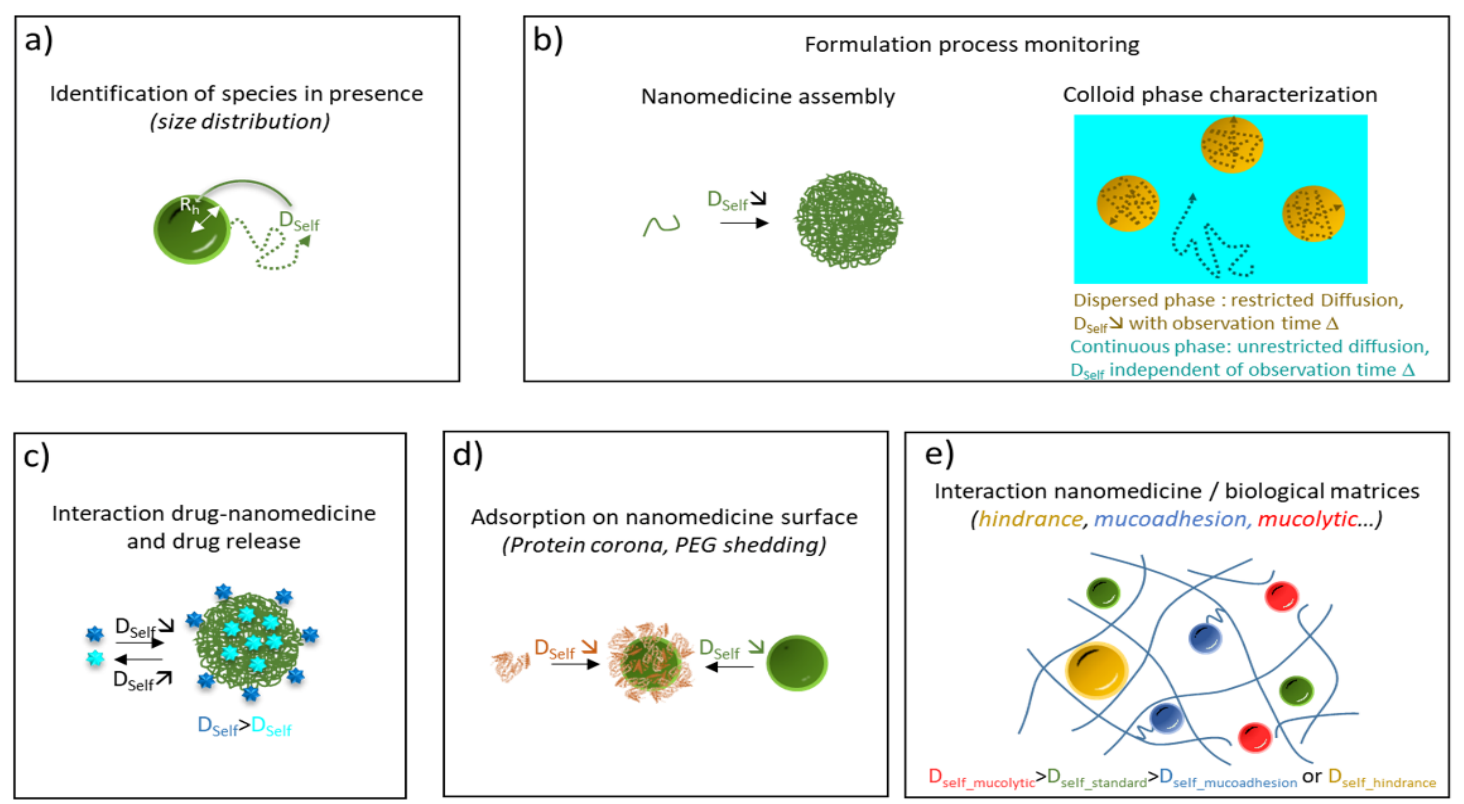

Fig. 6 Potentials of PFG-NMR to characterize nanomedicine in vitro: a Identification of species in presence based on size determination, $\boldsymbol{b}$ Formulation process monitoring such as assembly or colloid phase monitoring, $c$ interactions between drug and nanomedicine or drug release process, $\boldsymbol{d}$ monitoring of adsorption process of biological molecules on nanomedicine surface and $\boldsymbol{e}$ exploration of interaction mechanisms with biological matrices such as mucus (hindrance, mucoadhesion or mucolytics phenomena). 
as demonstrated for polymer micelles encapsulating anesthetic [66] or antiinflammatory [67] hydrophobic drugs.

When nanomedicine is dual drug loaded, the estimation of drug diffusion coefficients and fractions can unravel the impact of the formulation process on drug location. Simeonova et al. [68] have quantified the free and bound fractions of two drug signals, the antibiotic DAU and the anticancer 5-fluorouracil (5FU), using respectively ${ }^{1} \mathrm{H}$ and ${ }^{19} \mathrm{~F}$ PFG-NMR upon simultaneous or consecutive inclusion in polymeric nanoparticles. They showed that DAU bound fraction was higher for simultaneous inclusion than for consecutive ones while the fraction of bound 5FU rises when switching from simultaneous to consecutive inclusion. This highlights the drug nanoparticles' loading pattern. When the inclusion was simultaneous, nanoparticles were predominantly loaded with entrapped DAU with a low concentration of 5FU, and with the consecutive inclusion, the 5FU is entrapped inside the nanoparticle while DAU is weakly adsorbed on the surface.

The observation of drug diffusion coefficient evolution with the diffusion observation time $\Delta$, reflecting possible restrictions, can inform on the localization of the drug in a biphasic system. Mathias et al. [69] have studied the drug payload of a hydrogel of micelles. The hydrogel was loaded with a hydrophilic anticancer 5FU drug or its hydrophobic analog (1,3dimethyl-5-fluorouracil or DMFU). The diffusion coefficient measured for 5FU was larger than DMFU's one and the diffusion of DMFU was more hindered than 5FU's. This confirmed that DMFU was mainly in the micelle hydrophobic core with hindered diffusion while 5FU was mostly in the water phase with relatively free diffusion.

\subsubsection{Nanomedicine Drug Release}

To be efficient, nanomedicine will have to release its drug payload at the right time and the right place. In vitro, the mechanisms of drug release can be studied by measuring the impact of the release on the drug selfdiffusion coefficient or by performing mutual diffusion experiment to study drug release rates (Fig. 6c).

Responsive nanopharmaceuticals polymeric drug coatings understanding was increased by the PFG-NMR evaluation of the impact of microscopic structural modifications such as $\mathrm{H}$-bonding on potential drug macroscopic diffusion through a polyacrylate network[70].

The Alexander et al. study [39] is a good illustration of the impact of the release on the drug self-diffusion coefficient. They showed that the self-diffusion coefficients measured by PFG-NMR of the antiinflammatory flurbiprofen drug encapsulated in pluronic micelles evolved with $\mathrm{pH}$. Below $\mathrm{pH} 6.5$, the flurbiprofen diffusion coefficient is similar to the micelle one, meaning that the drug is solubilized within the micelles. But, when the $\mathrm{pH}$ is increased, the drug diffusion coefficient gradually increases and the fraction of drug still within the micelles diminishes, meaning that the drug is released.

If external stimuli such as $\mathrm{pH}$ modification can trigger the drug release, the way some nanomedicines interact with the biological membranes is also important to determine the drug release. PFG-NMR can contribute to differentiate the intracellular release of free from nanomedicine entrapped drugs as shown by Lopes et al. [71]. They studied the interaction of free 5FU and 5FU loaded polymeric nanoparticles with liposome phospholipid bilayer, as a model for biomembranes. Self-diffusion was estimated with PFG-NMR from the 5FU and the liposome ${ }^{1} \mathrm{H}$ NMR signals. As expected, the 5FU entrapped in the polymeric nanoparticle had a smaller diffusion coefficient than free 5FU due to the restricted environment. When mixed with liposomes, the diffusion of 5FU entrapped in polymeric nanoparticles 
increased while the free 5FU one decreased. Moreover, the liposome diffusion coefficient increased regardless of whether the drug was free or in the nanoparticles. This reflects, on one side, a concentrationdependent reversible diffusion for free $5 \mathrm{FU}$ due to a good membrane permeation but poor retention within the membrane and, on the other side, a sustained drug release from the nanoparticles adsorbed on the membrane surface based on the deeper drug penetration in the membrane.

Mutual diffusion study is another way to get information on drug release. In this case, drug release using labeling agents entrapped within the nanomedicine is monitored by spatially resolved NMR methods, either imaging or NMR profiling. However, most of the time only qualitative information is produced, mainly limited by the difficulty to convert signal modifications into concentration variations. For example, Kato et al. [72] have monitored drug release from liposomes loaded with dual magnetic labels, superparamagnetic iron oxide (SPIO) nanoparticles, and either Gadolinium chelate for MRI exploration or 5fluorouracil for ${ }^{19} \mathrm{~F}$ MRS. The size difference between the two labels generates different diffusion properties, the large SPIO diffusion being reduced compared to smaller Gadolinium chelates or fluorinated anticancer drugs. In this example, no mutual diffusion coefficient quantification was performed but still qualitative valuable information on drug release was obtained. When the two labels are nearby inside the liposome, the susceptibility effect of the superparamagnetic label is masking the effect of the second label. But, when liposomes release their content, labels start to diffuse, and the distance between both labels increases. The onset of the positive T1 image contrast of the Gadolinium chelate or the ${ }^{19} \mathrm{~F}$ signal enhancement due to resonance linewidth narrowing is therefore the reflection of how the drug has diffused out of the liposomes.

\subsubsection{Nanomedicine Surface Adsorption}

When administered in vivo, the interaction of the nanomedicine with the constituents of the biological fluids alters their physiochemical characteristics and therefore modify their circulation time in blood and their accumulation at the target site. The understanding of these interactions, the corona formation, and kinetics, is mandatory to understand their biological behavior and outcomes. PFGNMR can be applied to study in vitro the corona formation, made of proteins most of the time (Fig. 6d). Two options are possible. The first one is to monitor the increase of the nanomedicine hydrodynamic radius upon protein adsorption. In this case, the main issue is the accuracy of the measure as the radius growth will be limited. Furthermore, the nanomedicine NMR signal is usually low, due to strong T2 weighting inherent to weak object mobility and lost in the middle of the multiple ${ }^{1} \mathrm{H}$ signals issued from the complex biological environment. Carril et al. [73] have proposed an elegant way to overcome these issues by labeling nanomedicine with ${ }^{19} \mathrm{~F}$ and perform ${ }^{19} \mathrm{~F}$ self-diffusion coefficient measurement to evaluate changes in hydrodynamic radius. Nanomedicines were gold core nanoparticles of different sizes, labeled with fluorinated polyethylene glycol. The method was able to pick up the small increases of the hydrodynamic radius induced by protein adsorption both in a model environment with only one type of protein present and in a complex environment such as plasma or blood. The use of fluorine to simplify the NMR signal opens the door to future in vivo nanomedicine monitoring using MRS. The second option is to visualize the strong decrease of the self-diffusion coefficient of the proteins upon their adsorption on the nanomedicine. Again, this is not trivial as the protein ${ }^{1} \mathrm{H}$ NMR signal can drastically diminish when adsorbed on the nanomedicine due to the slow rotational diffusion of the nanomedicine [74]. Despite inherent low signal, Kato et al. [75] were 
able to show that bovine serum albumin (BSA) in the presence of fullerene colloidal particles, presents two diffusion coefficients, a fast diffusing one corresponding to bulk BSA and a slow diffusing one assigned to strongly adsorbed BSA molecule onto particles. The number of BSA molecules bound to fullerene colloidal particles was even evaluated to less than $5 \%$ indicating that most of the BSA molecules diffuse freely in the fullerene colloidal suspensions.

To limit these interactions with biomolecules and enhance nanomedicine efficacy by reducing clearance by the reticuloendothelial system, steric stabilizers such as poly(ethylene glycol), PEG, can be anchored at the surface of nanomedicines. However, this coating can negatively impact the cellular uptake and must therefore be removed before reaching the target cells. PFG-NMR using the PEG ${ }^{1} \mathrm{H}$ NMR signal has proven to be a powerful tool to study the PEG shedding rate as demonstrated by the study implying small interfering ribonucleic acid encapsulated lipid nanoparticles in biofluids [76]. To monitor nanoparticles' surface coating shedding when in contact with serum, the PEG-lipid self-diffusion of free or associated to nanoparticles was studied. The authors showed that PEG with a smaller lipid tail shed faster than those with a long tail as the result of a reduced contact between the lipid and the membrane.

\subsubsection{Interaction of Nanomedicine With Biological Barrier}

Nanomedicines, especially those for oral drug delivery, will often have to cross several biological barriers (mucus, interstitial matrix, membrane...) to reach their biotarget. One of these is the mucus layer protecting many epithelial surfaces such as the gastrointestinal tract, lungs, eyes, or the vagina. The mucus is a semipermeable viscoelastic hydrogel composed mainly of water and in a minor proportion of glycoproteins (mucin), lipids, and salt. The ability of nanomedicines to cross the mucus layer will depend on both their trapping in the mucus by hindrance or adhesion and the mucus clearance turnover. Tools to evaluate interactions between nanomedicines and mucus are therefore crucial for the development of more efficient drug delivery systems. Even if only a few studies have been published, PFG-NMR offers a unique insight into the study of mucus structure and mucus/nanomedicine interactions (Fig. 6e).

\subsubsection{Probing the Hydrogel Structure}

The dynamics and structure of the complex biological matrices can be studied by PFGNMR. The addition of probe molecule or nanoparticle to assess the submicron structure of hydrogel networks was described by de Kort et al. [46] in a review applied to food biopolymers. This method was also applied with benefice to biological barrier exploration as demonstrated by the study of the influence of $\mathrm{pH}$ on the mucin gel structure using PEG molecules as probes [77]. As the decrease in $\mathrm{pH}$ is meant to modify the mucin charge density, resultant matrix structure modifications were expected. When probed by intermediate size PEGs, the probe diffusion coefficient presented a minimum at intermediate $\mathrm{pH}$ reflecting a transport through a structured three-dimensional mucin network promoted by the hydrophobic interactions. At low and high $\mathrm{pH}$, the probe diffusion increases, due to respectively a higher network heterogeneity with polymer-rich clusters and water-rich domains and increased network flexibility favored by weaker hydrophobic interactions and strong electrostatic repulsion between mucin molecules. However, the matrix structure unraveled depends on the adequacy between the probe size and flexibility, the mesh pore size, and the diffusion observation time $\Delta$. Indeed, when larger PEGs were used as a probe, diffusion coefficients showed a continuous increase with decreasing $\mathrm{pH}$, indicating a loss in the network homogeneity. The network 
flexibility observed at high $\mathrm{pH}$ facilitates the transport of intermediate PEGs but did not affect larger PEGs.

\subsubsection{Interaction of Nanomedicine With Hydrogel}

In vivo, one of the main transport mechanisms of nanomedicine through the biological matrix is passive diffusion. So, in vitro self-diffusion or mutual diffusion experiments are particularly relevant to understand and optimize nanomedicine barrier crossing and check the adequacy between the nanomedicine and the matrix as illustrated by the following examples.

Lafitte et al. [78] have studied by PFGNMR the impact of the matrix nature on micelles' self-diffusion. In mucin, only hindrance effects by an obstruction are observed while in mucus the micelle diffusion is further slowed down due to interactions with lipids of the matrix. Wisniewska et al. [79] have also studied micelle diffusion but under nonequilibrium conditions, from a surfactant solution to a polymeric hydrogel, and with both mutual and self-diffusion using NMR spectrometers. Each method sheds a different light on the transport mechanisms. Mutual diffusion coefficients were determined from the surfactant concentration profile, itself determined by one dimension chemical shift imaging (CSI) signal profiles using Fick's second law (Equation 3). Surfactant self-diffusion coefficients were determined by slice selective PFG-NMR in specific positions located in the solution and the gel. They showed that the surfactant self-diffusion in the hydrogel decreases until a plateau value when surfactant concentration increases while in the solution it remains constant. The surfactant concentration plays a key role. Below the critical micellar concentration, the surfactant diffuses as unimers whereas, over this limit, surfactant self-assembles into micelles. If surfactant unimers freely diffuse into the mesh, micellar diffusion is hindered by the polymeric network. The ratio between the self-diffusion and the mutual surfactant diffusion coefficient was around 0.8 reflecting the difference of length and time exploration scale between both methods. The mutual diffusion gives information on the macroscopic scale and on a large time scale while self-diffusion informs on the surfactant assembly state (unimer or micelle) and its interaction with the polymer network on a much shorter time scale.

Wang et al. [80] have also illustrated the interest of mutual diffusion to study nanomedicine penetration rate in a hydrogel. The nanomedicine label, in this case, is not provided by the spectral separation as for Wisniewska et al. [79] but by incorporating a contrast agent in the polymeric nanoparticles, either a gadolinium chelate as paramagnetic contrast agent or magnetite nanoparticles as superparamagnetic agent. The penetration of the contrast agent from the nanomedicines solution into the hydrogel was quantified versus time by MRI image contrast modifications. They were able to quantify diffusion rate variations depending on the steric hindrance using different hydrogel polymer content or on the electrostatic interaction by varying the nanoparticles or hydrogel charges.

Several strategies have been proposed to modify the surface of nanomedicine to improve transport through mucosal tissues, including mucoadhesion, mucopenetration, or mucolytics, and once again, PFG-NMR can help to quantify their efficacy. The ability of mucolytic decorated nanomedicine to break up the mucin structure was demonstrated by Pereira de Sousa et al. [81] who showed an important increase of mucin mobility when enzyme functionalized nanomedicine was diffusing in. Another way to see the mucus modifications induced by the mucoactive coating of the nanomedicine is to probe the mucus hydration temporal and spatial modifications by measuring the water selfdiffusion coefficient using diffusion 
weighted-NMR profiling or MRI. Even if their study did not imply nanomedicine, Marshall et al. [82] demonstrated the feasibility of this approach.

\subsubsection{Bacterial Biofilm}

Another kind of biological barrier that nanomedicine may have to cross is bacterial biofilms. The penetration ability of nanomedicine in bacterial biofilms is one of the keys to develop efficient antibacterial nanomedicine. Kwak et al. [83] have studied by PFG-NMR the diffusion properties of surfactant micelles in polysaccharide gel to provide information on their interaction that can favor or limit biofilm internalization. The micelle size and the polysaccharide concentration were found to hinder the diffusion and compromise antibacterial activity. The characterization of the biofilm structure and heterogeneity can also be studied using water DW-MRI [84] or water PFG-NMR [85] alone or coupled with $\mathrm{T} 2$ relaxation measurement to obtain a D-T2 map. The mutual diffusion of a paramagnetic contrast agent in a biofilm mass has also been studied [86] by MRI and could be transposed to nanomedicine study.

\subsection{In vivo Nanomedicine Fate}

In vivo nanomedicine fate monitoring would the grail. Transposition to in vivo of the NMR diffusometry methods implemented in vitro would be very attractive. However, only a few works were reported in the literature because in vivo nanomedicine fate is very challenging.

Transposition of the PFG-NMR method in vivo relies on diffusion-weighted magnetic resonance spectroscopy, DW-MRS, to acquire a series of localized diffusionweighted spectra to estimate self-diffusion coefficients. Potentially, nanomedicine integrity or binding evolution monitoring, drug release tracking or biological matrices, and nanomedicines interactions characterization could become feasible.
However, in vivo, everything is more complicated. The water signal is massive and needs to be suppressed, biological motions such as respiration, heart beat, or bulk motion can compromise the spectra quality and their influences must be limited or taken into account by synchronizing the acquisition. The voxel volume and localization need to be well adapted to the anatomy to be large enough for an acceptable signal-to-noise ratio while only encompassing homogenous tissue to be meaningful. The idea is to be able to measure non-water self-diffusion coefficients to probe nanomedicine (size, integrity evolution, binding...), their environment structure (complex biological matrix) or their drug payload, using a specific NMR signal of the nanomedicine, of the drug or the complex matrix and if possible, without the use of an exogenous probe molecule. This is very ambitious. Indeed, even if a lot of precautions are taken, the ${ }^{1} \mathrm{H}$ NMR signal of interest will have a high chance to be like a needle in a haystack. However, the feasibility of in vivo non-water self-diffusion measurement by DW-MRS has been demonstrated in biological tissues [87] both for intramyocellular [88] or intratumoral [89] lipid droplet sizing or brain microstructures study using metabolites self-diffusion [90,91]. The characterization of the extracellular matrix degradation in intervertebral disc degeneration has also been explored, but ex vivo [92]. Once again, an interesting way to simplify the spectrum could be to switch to a fluorine-19 signal which offers a specific NMR signature and no inherent tissue background signal. Using ${ }^{19} \mathrm{~F}$ DW-MRS for nanomedicine evaluation, Waters et al. [93] were able to detect binding of angiogenesis-targeted perfluorocarbon nanoparticles in vivo. We also demonstrated the feasibility of in vivo perfluorocarbon loaded lipid nanocapsules self-diffusion coefficient estimation using ${ }^{19} \mathrm{~F}$ DW-MRS, accumulated in the mouse liver after intravenous injection as shown in figure 7 . 


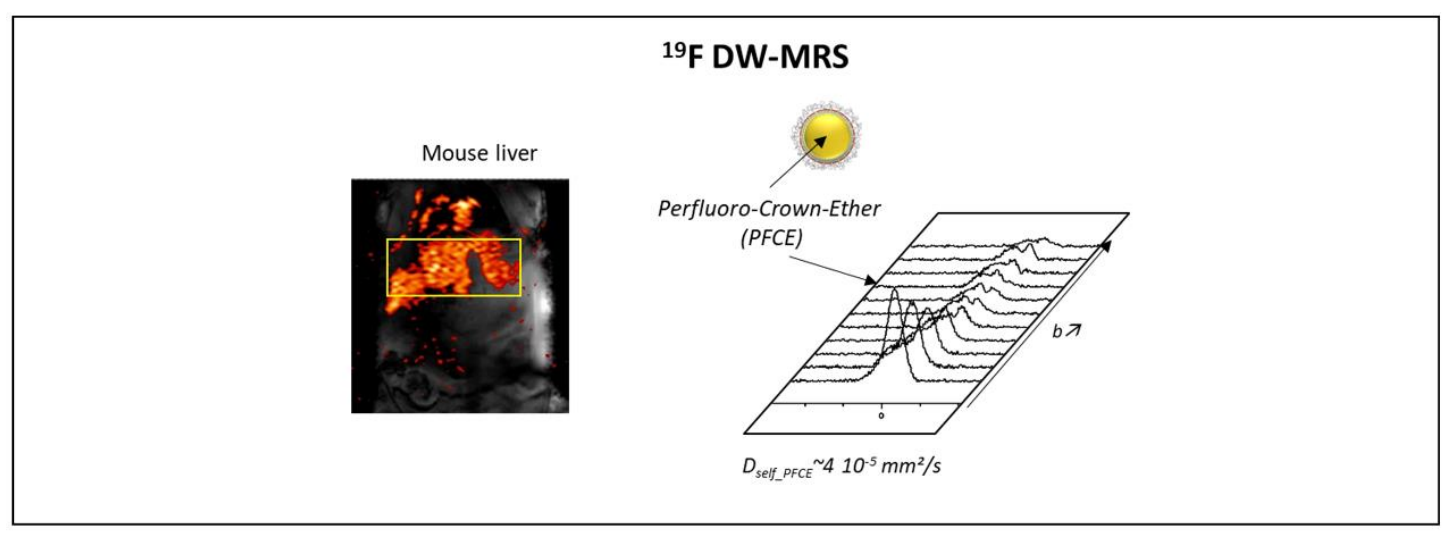

Fig. 7 The feasibility of in vivo fate monitoring using ${ }^{19} F$ DW-MRS was demonstrated for perfluorocarbon-loaded lipid nanocapsules injected intravenously $(700 \mu L)$ in a mouse. As expected, nanomedicines accumulate in the liver, and nanomedicine self-diffusion coefficient evaluation was possible in vivo [Unpublished work from L. Lemaire].

The transposition in vivo of the mutual diffusion experiment to probe nanomedicine transport or drug release or uptake would be attractive. However, in vivo, the quantification of mutual diffusion coefficient seems very challenging and most of the time only qualitative monitoring is obtained. Furthermore, pure diffusive effects could be difficult to be distinguished from vascular effects. Nevertheless, monitoring the distribution of therapeutic nanomedicine or drugs in an organ is important to improve response prediction or adapt treatment. In vitro, NMR profiling or MRI spatial nanomedicine tracking has been performed using contrast agents incorporated in nanomedicine as illustrated by the following studies. De Smet et al. [94] and Tagami et al. [95] have studied the drug release of co-loaded doxorubicin drug and paramagnetic Gadolinium-based agent temperature sensitive liposomes in rodent tumor models. At physiological temperature, the longitudinal relaxivity of the contrast agent is low due to the limited liposome transmembrane water exchange rate. They showed that upon mild hyperthermia, the contrast agent is released and the observed $\mathrm{T} 1$ locally decrease on $\mathrm{T} 1$ map, correlated to gadolinium or drug concentrations. Wang et al. [96] monitored in vivo in a rodent tumor model the drug release from redox-responsive polymeric magnetosomes encapsulating SPIO. The co-encapsulation of the hydrophobic doxorubicin drug was shown to modify the T2 contrast by repelling the water molecules from the SPIO. The modification of T2 contrast allowed to monitor the drug release. Onuki et al. [97] have extended in vivo the in vitro study of Kato et al. [72] to monitor the drug release profile of nanospheres. Besides the anticancer drug 5FU, the carrier was also loaded with two contrast agents, superparamagnetic iron oxide nanoparticles and Gadolinium chelates to distinguish the released drug from encapsulated one. When the carrier releases its payload, the small Gadolinium chelates diffuse faster than the large SPIO nanoparticles. And when the Gadolinium chelates are far enough from the susceptibility effect of the SPIO, its positive contrast enhancement effect is expressed on the image, signing of the drug release. They demonstrated the feasibility of in vivo drug release from carrier monitoring in a mice tumor model using both $\mathrm{T} 2 *$ weighted images for SPIO visualization and T1 mapping to quantify Gadolinium chelates concentration. However, they pointed out that the release profile of the paramagnetic 
contrast agent was not strictly similar to that of the drug in vivo. Therefore, a direct detection of the drug using ${ }^{19} \mathrm{~F}$ MRS would be better but challenging in term of sensitivity.

\section{Conclusions and Future Directions}

This article has surveyed the potential of NMR diffusometry for nanomedicine applications. Several valuable examples were presented to highlight method inputs to the nanomedicine field. Many other interesting studies would certainly deserve to be mentioned in this review, but the aim was not to be exhaustive but rather illustrative of the potential of the method. NMR diffusometry demonstrated to be an in vitro tool that can be applied routinely to the nanomedicine field as a complementary analysis method. The strength of NMR diffusometry mainly relies on its unique ability to offer a characterization continuum from in vitro to in vivo by transposing in vitro methods to in vivo dedicated systems using either localized spectroscopy or imaging. Surely, with the recent large use of nanoparticle-based vaccines in the fight against the COVID-19 pandemic, the development of nanomedicines in the coming years should greatly accelerate and new tools will be needed to investigate their fate in bioenvironments. In vivo NMR diffusometry applications are yet in their early development stage but should contribute to better understand complex interaction with biological systems. On one side, diffusion-weighted localized spectroscopy has shown that in vivo access to self-diffusion properties of ${ }^{1} \mathrm{H}$ nuclei signals from molecules other than only water is possible. If a specific nanomedicine or drug moieties signal can be picked up, this opens possibilities to probe nanomedicine integrity or drug delivery. Clearly, compromises will have to be done to comply with in vivo requirements (small b values to keep reasonable scanning duration, lower maximum $b$ value to accommodate MRI systems gradients limits...) and diffusion coefficient measures will certainly not reach in vitro precision standards. Still, crucial information could be obtained, especially in the longitudinal monitoring of nanomedicine fate. On the other side, MRI has greatly demonstrated its ability to image and monitor spatial and temporal signal evolutions. If nanomedicines are made MR visible by contrast agent labeling, mutual diffusion effect can be used to monitor in vivo nanomedicine tracking or drug release. However, some issues are recurrent: Does the co-localization of the label, the nanomedicine, and the drug last the whole experiment duration? How to convert signal contrast modifications in terms of concentrations? Is it toxic? However, in the light of the technological developments of MRI systems (higher sensitivity offered by the high magnetic field or cryogenic coil, higher magnetic gradients...) and the development of new contrast agents [98100], we believe that in vivo transposition is possible if not straightforward and will offer unique opportunity to better understand nanomedicine in vivo fate.

\section{Acknowledgments}

Samuel Bonnet was financially supported by Biogenouest at the University of Angers (http://www.biogenouest.org). 


\section{References}

[1] V. Gadekar, Y. Borade, S. Kannaujia, K. Rajpoot, N. Anup, V. Tambe, K. Kalia, R.K. Tekade, Nanomedicines accessible in the market for clinical interventions, Journal of Controlled Release. 330 (2021) 372-397. https://doi.org/10.1016/j.jconrel.2020.12.034.

[2] K. Park, The drug delivery field at the inflection point: Time to fight its way out of the egg, Journal of Controlled Release. 267 (2017)

$2-14$. https://doi.org/10.1016/j.jconrel.2017.07.030.

[3] K. Greish, A. Mathur, M. Bakhiet, S. Taurin, Nanomedicine: is it lost in translation?, Therapeutic Delivery. 9 (2018) 269-285. https://doi.org/10.4155/tde-2017-0118.

[4] S. Gioria, F. Caputo, P. Urbán, C.M. Maguire, S. Bremer-Hoffmann, A. Prina-Mello, L. Calzolai, D. Mehn, Are existing standard methods suitable for the evaluation of nanomedicines: some case studies, Nanomedicine. 13 (2018) 539-554. https://doi.org/10.2217/nnm-2017-0338.

[5] J.B. Hall, M.A. Dobrovolskaia, A.K. Patri, S.E. McNeil, Characterization of nanoparticles for therapeutics, Nanomedicine. 2 (2007) 789-803. https://doi.org/10.2217/17435889.2.6.789.

[6] F. Caputo, J. Clogston, L. Calzolai, M. Rösslein, A. Prina-Mello, Measuring particle size distribution of nanoparticle enabled medicinal products, the joint view of EUNCL and NCI-NCL. A step-by-step approach combining orthogonal measurements with increasing complexity, Journal of Controlled Release. $299 \quad$ (2019) 31-43. https://doi.org/10.1016/j.jconrel.2019.02.030.

[7] J.-B. Coty, C. Vauthier, Characterization of nanomedicines: A reflection on a field under construction needed for clinical translation success, Journal of Controlled Release. 275 (2018) 254-268. https://doi.org/10.1016/j.jconrel.2018.02.013.

[8] C.M. Maguire, M. Rösslein, P. Wick, A. Prina-Mello, Characterisation of particles in solution - a perspective on light scattering and comparative technologies, Science and Technology of Advanced Materials. 19 (2018) 732-745.

https://doi.org/10.1080/14686996.2018.1517 587.

[9] F. Varenne, A. Makky, M. Gaucher-Delmas, F. Violleau, C. Vauthier, Multimodal Dispersion of Nanoparticles: A Comprehensive Evaluation of Size Distribution with 9 Size Measurement Methods, Pharmaceutical Research. 33 (2016) 1220-1234. https://doi.org/10.1007/s11095016-1867-7.
[10] ISO 22412:2017, Particle Size Analysis Dynamic Light Scattering (DLS), ISO, 2017., (n.d.).

[11] K. Fischer, M. Schmidt, Pitfalls and novel applications of particle sizing by dynamic light scattering, Biomaterials. 98 (2016) 7991.

https://doi.org/10.1016/j.biomaterials.2016.0 5.003 .

[12] O. Glatter, Scattering Methods and their Application in Colloid and Interface Science, Elsevier, 2018. https://doi.org/10.1016/C2016-0-04640-5.

[13] N. Lorén, J. Hagman, J.K. Jonasson, H. Deschout, D. Bernin, F. Cella-Zanacchi, A. Diaspro, J.G. McNally, M. Ameloot, N. Smisdom, M. Nydén, A.-M. Hermansson, M. Rudemo, K. Braeckmans, Fluorescence recovery after photobleaching in material and life sciences: putting theory into practice, Quarterly Reviews of Biophysics. 48 (2015) 323-387. https://doi.org/10.1017/S0033583515000013.

[14] H. Deschout, K. Raemdonck, J. Demeester, S.C. De Smedt, K. Braeckmans, FRAP in Pharmaceutical Research: Practical Guidelines and Applications in Drug Delivery, Pharmaceutical Research. 31 (2014) 255-270. https://doi.org/10.1007/s11095-013-1146-9.

[15] G. Colafemmina, H. Mateos, G. Palazzo, Diffusion NMR studies of complex liquid formulations, Current Opinion in Colloid \& Interface Science. 48 (2020) 109-120. https://doi.org/10.1016/j.cocis.2020.04.006.

[16] P.E. Petrochenko, N. Pavurala, Y. Wu, S. Yee Wong, H. Parhiz, K. Chen, S.M. Patil, H. Qu, P. Buoniconti, A. Muhammad, S. Choi, D. Kozak, M. Ashraf, C.N. Cruz, J. Zheng, X. Xu, Analytical considerations for measuring the globule size distribution of cyclosporine ophthalmic emulsions, International Journal of Pharmaceutics. 550 (2018) 229-239. https://doi.org/10.1016/j.ijpharm.2018.08.030.

[17] N. Bertrand, J.-C. Leroux, The journey of a drug-carrier in the body: An anatomophysiological perspective, Journal of Controlled Release. 161 (2012) 152-163. https://doi.org/10.1016/j.jconrel.2011.09.098.

[18] X. Murgia, B. Loretz, O. Hartwig, M. Hittinger, C.-M. Lehr, The role of mucus on drug transport and its potential to affect therapeutic outcomes, Advanced Drug Delivery Reviews. 124 (2018) 82-97. https://doi.org/10.1016/j.addr.2017.10.009.

[19] J.K.G. Dhont, An introduction to dynamics of colloids. Studies in interface science, Möebius and R. Miller, Elsevier, 1996.

[20] R. Bowen, A. Mongruel, Calculation of the collective diffusion coefficient of electrostatically stabilised colloidal particles, 
Colloids and Surface A : Physicochemical and Engineering Aspects. (1998) 161-172.

[21] M. Tokuyama, I. Oppenheim, Dynamics of hard-sphere suspensions, Physical Review E. $50 \quad$ (1994) R16-R19. https://doi.org/10.1103/PhysRevE.50.R16.

[22] P. Occhipinti, P.C. Griffiths, Quantifying diffusion in mucosal systems by pulsedgradient spin-echo NMR, Advanced Drug Delivery Reviews. 60 (2008) 1570-1582. https://doi.org/10.1016/j.addr.2008.08.006.

[23] J. Appell, G. Porte, E. Buhler, Self-Diffusion and Collective Diffusion of Charged Colloids Studied by Dynamic Light Scattering, The Journal of Physical Chemistry B. 109 (2005) 13186-13194. https://doi.org/10.1021/jp051016k.

[24] U. Zettl, S.T. Hoffmann, F. Koberling, G. Krausch, J. Enderlein, L. Harnau, M. Ballauff, Self-Diffusion and Cooperative Diffusion in Semidilute Polymer Solutions As Measured by Fluorescence Correlation Spectroscopy, Macromolecules. 42 (2009) 9537-9547. https://doi.org/10.1021/ma901404g.

[25] J. Crank, The mathematics of diffusion, 2nd ed., Clarendon Press, Oxford, 1975.

[26] E.O. Stejskal, J.E. Tanner, Spin Diffusion Measurements: Spin Echoes in the Presence of a Time-Dependent Field Gradient, The Journal of Chemical Physics. 42 (1965) 288292. https://doi.org/10.1063/1.1695690.

[27] P. Mansfield, Multi-planar image formation using NMR spin echoes, Journal of Physics C: Solid State Physics. 10 (1977) L55-L58. https://doi.org/10.1088/0022-3719/10/3/004.

[28] M.A. Miller, S. Gadde, C. Pfirschke, C. Engblom, M.M. Sprachman, R.H. Kohler, K.S. Yang, A.M. Laughney, G. Wojtkiewicz, N. Kamaly, S. Bhonagiri, M.J. Pittet, O.C. Farokhzad, R. Weissleder, Predicting therapeutic nanomedicine efficacy using a companion magnetic resonance imaging nanoparticle, Science Translational Medicine. $7 \quad$ (2015) 314ra183-314ra183. https://doi.org/10.1126/scitranslmed.aac6522.

[29] F. Reeßing, W. Szymanski, Following nanomedicine activation with magnetic resonance imaging: why, how, and what's next?, Current Opinion in Biotechnology. 58 (2019)

$9-18$. https://doi.org/10.1016/j.copbio.2018.10.008.

[30] J. Nel, F. Franconi, N. Joudiou, P. Saulnier, B. Gallez, L. Lemaire, Lipid nanocapsules as in vivo oxygen sensors using magnetic resonance imaging, Materials Science and Engineering: C. 101 (2019) 396-403. https://doi.org/10.1016/j.msec.2019.03.104.

[31] L. Lemaire, G. Bastiat, F. Franconi, N. Lautram, T. Duong Thi Dan, E. Garcion, P. Saulnier, J.P. Benoit, Perfluorocarbon-loaded lipid nanocapsules as oxygen sensors for tumor tissue pO2 assessment, European Journal of Pharmaceutics and Biopharmaceutics. 84 (2013) 479-486. https://doi.org/10.1016/j.ejpb.2013.01.003.

[32] F. Franconi, L. Lemaire, B. Siegler, J.-C. Gimel, P. Saulnier, NMR diffusometry data sampling optimization for mixture analysis, Journal of Pharmaceutical and Biomedical Analysis. $148 \quad$ (2018) 156-162. https://doi.org/10.1016/j.jpba.2017.09.028.

[33] M. Holz, S.R. Heil, A. Sacco, Temperaturedependent self-diffusion coefficients of water and six selected molecular liquids for calibration in accurate $1 \mathrm{H}$ NMR PFG measurements, Physical Chemistry Chemical Physics. 2 (2000) 4740-4742. https://doi.org/10.1039/b005319h.

[34] S.W. Provencher, CONTIN: A general purpose constrained regularization program for inverting noisy linear algebraic and integral equations, Computer Physics Communications. 27 (1982) 229-242. https://doi.org/10.1016/0010-4655(82)901746.

[35] W. Windig, B. Antalek, Direct exponential curve resolution algorithm (DECRA): A novel application of the generalized rank annihilation method for a single spectral mixture data set with exponentially decaying contribution profiles, Chemometrics and Intelligent Laboratory Systems. 37 (1997) 241-254. https://doi.org/10.1016/S01697439(97)00028-2.

[36] M. Nilsson, G.A. Morris, Speedy Component Resolution: An Improved Tool for Processing Diffusion-Ordered Spectroscopy Data, Analytical Chemistry. 80 (2008) 3777-3782. https://doi.org/10.1021/ac7025833.

[37] L. Castañar, G.D. Poggetto, A.A. Colbourne, G.A. Morris, M. Nilsson, The GNAT: A new tool for processing NMR data, Magnetic Resonance in Chemistry. 56 (2018) 546-558. https://doi.org/10.1002/mrc.4717.

[38] O. Söderman, H. Walderhaug, Micellar Solutions and Microemulsions, in: R.K. Harris (Ed.), Encyclopedia of Magnetic Resonance, John Wiley \& Sons, Ltd, Chichester, UK, 2011. https://doi.org/10.1002/9780470034590.emrs tm0310.pub2.

[39] S. Alexander, W.M. de Vos, T.C. Castle, T. Cosgrove, S.W. Prescott, Growth and Shrinkage of Pluronic Micelles by Uptake and Release of Flurbiprofen: Variation of $\mathrm{pH}$, Langmuir. 28 (2012) 6539-6545. https://doi.org/10.1021/la204262w.

[40] B. Antalek, Accounting for Spin Relaxation in Quantitative Pulse Gradient Spin Echo NMR Mixture Analysis, Journal of the American Chemical Society. 128 (2006) 8402-8403. https://doi.org/10.1021/ja062592c. 
[41] C. Barrère, P. Thureau, A. Thévand, S. Viel, Acquisition strategy to obtain quantitative diffusion NMR data, Journal of Magnetic Resonance. $216 \quad$ (2012) 201-208. https://doi.org/10.1016/j.jmr.2011.12.022.

[42] P.N. Sen, Time-dependent diffusion coefficient as a probe of geometry, Concepts in Magnetic Resonance. 23A (2004) 1-21. https://doi.org/10.1002/cmr.a.20017.

[43] S. Babu, J.C. Gimel, T. Nicolai, Tracer Diffusion in Colloidal Gels, J. Phys. Chem. B. $112 \quad$ (2008) 743-748. https://doi.org/10.1021/jp076342+.

[44] J.-C. Gimel, T. Nicolai, Self-diffusion of noninteracting hard spheres in particle gels, Journal of Physics: Condensed Matter. 23 (2011) 234115. https://doi.org/10.1088/09538984/23/23/234115.

[45] C. Mayer, NMR Characterization Techniques - Application to Nanoscaled Pharmaceutical Carriers, in: C.S.S.R. Kumar (Ed.), Nanotechnologies for the Life Sciences, Wiley-VCH Verlag GmbH \& Co. KGaA, Weinheim, Germany, 2007. https://doi.org/10.1002/9783527610419.ntls0 027.

[46] D.W. de Kort, J.P.M. van Duynhoven, H. Van As, F. Mariette, Nanoparticle diffusometry for quantitative assessment of submicron structure in food biopolymer networks, Trends in Food Science \& Technology. $42 \quad$ (2015) 13-26. https://doi.org/10.1016/j.tifs.2014.11.003.

[47] Y. Cu, W.M. Saltzman, Mathematical modeling of molecular diffusion through mucus, Advanced Drug Delivery Reviews. 61 (2009) 101-114. https://doi.org/10.1016/j.addr.2008.09.006.

[48] L. Masaro, X.X. Zhu, Physical models of diffusion for polymer solutions, gels and solids, Progress in Polymer Science. 24 (1999) 731-775. https://doi.org/10.1016/S00796700(99)00016-7.

[49] J.H. Jensen, J.A. Helpern, MRI quantification of non-Gaussian water diffusion by kurtosis analysis, NMR in Biomedicine. 23 (2010) 698-710. https://doi.org/10.1002/nbm.1518.

[50] J.H. Jensen, J.A. Helpern, A. Ramani, H. Lu, K. Kaczynski, Diffusional kurtosis imaging: The quantification of non-Gaussian water diffusion by means of magnetic resonance imaging, Magnetic Resonance in Medicine. 53 (2005)

1432-1440. https://doi.org/10.1002/mrm.20508.

[51] A.G. Cherstvy, S. Thapa, C.E. Wagner, R. Metzler, non-Gaussian, non-ergodic, and nonFickian diffusion of tracers in mucin hydrogels, Soft Matter. 15 (2019) 2526-2551. https://doi.org/10.1039/C8SM02096E.

[52] S. Murgia, P. Fadda, G. Colafemmina, R. Angelico, L. Corrado, P. Lazzari, M.
Monduzzi, G. Palazzo, Characterization of the Solutol ${ }^{\circledR}$ HS15/water phase diagram and the impact of the $\Delta$ 9-tetrahydrocannabinol solubilization, Journal of Colloid and Interface Science. 390 (2013) 129-136. https://doi.org/10.1016/j.jcis.2012.08.068.

[53] S.J. Law, M.M. Britton, Sizing of Reverse Micelles in Microemulsions using NMR Measurements of Diffusion, Langmuir. 28 (2012)

11699-11706. https://doi.org/10.1021/la300796u.

[54] G. Canzi, A.A. Mrse, C.P. Kubiak, DiffusionOrdered NMR Spectroscopy as a Reliable Alternative to TEM for Determining the Size of Gold Nanoparticles in Organic Solutions, The Journal of Physical Chemistry C. 115 (2011) 7972-7978. https://doi.org/10.1021/jp2008557.

[55] L. Guyon, E. Lepeltier, J.-C. Gimel, B. Calvignac, F. Franconi, N. Lautram, A. Dupont, C. Bourgaux, P. Pigeon, P. Saulnier, G. Jaouen, C. Passirani, Importance of Combining Advanced Particle Size Analysis Techniques To Characterize Cell-Penetrating Peptide-Ferrocifen Self-Assemblies, The Journal of Physical Chemistry Letters. 10 (2019) 6613-6620. https://doi.org/10.1021/acs.jpclett.9b01493.

[56] M. Garcia-Fuentes, D. Torres, M. MartínPastor, M.J. Alonso, Application of NMR Spectroscopy to the Characterization of PEGStabilized Lipid Nanoparticles, Langmuir. 20 (2004) 8839-8845. https://doi.org/10.1021/la049505j.

[57] M. Valentini, A. Vaccaro, A. Rehor, A. Napoli, J.A. Hubbell, N. Tirelli, Diffusion NMR Spectroscopy for the Characterization of the Size and Interactions of Colloidal Matter: The Case of Vesicles and Nanoparticles, Journal of the American Chemical Society. 126 (2004) 2142-2147. https://doi.org/10.1021/ja037247r.

[58] S. Vázquez-Leyva, L. Vallejo-Castillo, C.A. López-Morales, J.E. Herbert-Pucheta, L.G. Zepeda-Vallejo, M. Velasco-Velázquez, L. Pavón, S.M. Pérez-Tapia, E. Medina-Rivero, Identity Profiling of Complex Mixtures of Peptide Products by Structural and Mass Mobility Orthogonal Analysis, Analytical Chemistry. $91 \quad$ (2019) 14392-14400. https://doi.org/10.1021/acs.analchem.9b0287 3.

[59] J. Ma, C. Guo, Y. Tang, J. Xiang, S. Chen, J. Wang, H. Liu, Micellization in aqueous solution of an ethylene oxide-propylene oxide triblock copolymer, investigated with $1 \mathrm{H}$ NMR spectroscopy, pulsed-field gradient NMR, and NMR relaxation, Journal of Colloid and Interface Science. 312 (2007) 390-396. https://doi.org/10.1016/j.jcis.2007.03.013. 
[60] X. Li, D.F. Shantz, PFG NMR studies of lysine-silica solutions, Journal of Colloid and Interface Science. 383 (2012) 19-27. https://doi.org/10.1016/j.jcis.2012.06.009.

[61] C. D’Agostino, V. Preziosi, A. Khan, M. Mantle, E. Fridjonsson, S. Guido, Microstructure evolution during nanoemulsification by NMR and microscopy, Journal of Colloid and Interface Science. 551 (2019) $138-146$. https://doi.org/10.1016/j.jcis.2019.04.098

[62] A. Buczkowski, M. Gorzkiewicz, A. Stepniak, M. Malinowska-Michalak, P. Tokarz, P. Urbaniak, M. Ionov, B. Klajnert-Maculewicz, B. Palecz, Physicochemical and in vitro cytotoxicity studies of inclusion complex between gemcitabine and cucurbit[7]uril host, Bioorganic Chemistry. 99 (2020) 103843. https://doi.org/10.1016/j.bioorg.2020.103843.

[63] A. Buczkowski, P. Tokarz, B. Palecz, Thermodynamic study of ethanol impact on gemcitabine binding to cucurbit[7]uril in aqueous solutions, The Journal of Chemical Thermodynamics. $153 \quad$ (2021) 106317. https://doi.org/10.1016/j.jct.2020.106317.

[64] M. Kühne, H. Lindemann, C. Grune, D. Schröder, Z. Cseresnyés, M. Godmann, A. Koschella, M.T. Figge, C. Eggeling, D. Fischer, T. Heinze, T. Heinzel, Biocompatible sulfated valproic acid-coupled polysaccharide-based nanocarriers with HDAC inhibitory activity, Journal of Controlled Release. 329 (2021) 717-730. https://doi.org/10.1016/j.jconrel.2020.10.006.

[65] G. Ivanova, M. Simeonova, E.J. Cabrita, M. Rangel, NMR Insight into the Supramolecular Structure of Daunorubicin Loaded Polymer Nanoparticles, The Journal of Physical Chemistry B. $115 \quad$ (2011) 902-909. https://doi.org/10.1021/jp109738e.

[66] K.I. Momot, P.W. Kuchel, Pulsed field gradient nuclear magnetic resonance as a tool for studying drug delivery systems, Concepts in Magnetic Resonance. 19A (2003) 51-64. https://doi.org/10.1002/cmr.a.10092.

[67] S. Alexander, T. Cosgrove, S.W. Prescott, T.C. Castle, Flurbiprofen Encapsulation Using Pluronic Triblock Copolymers, Langmuir. 27 (2011) 8054-8060. https://doi.org/10.1021/la201124c.

[68] M. Simeonova, M. Rangel, G. Ivanova, NMR study of the supramolecular structure of dual drug-loaded poly(butylcyanoacrylate) nanoparticles, Physical Chemistry Chemical $\begin{array}{llll}\text { Physics. } & 15 & \text { (2013) } & 16657 .\end{array}$ https://doi.org/10.1039/c3cp51471d.

[69] E.V. Mathias, J. Aponte, J.A. Kornfield, Y. Ba, Properties of small molecular drug loading and diffusion in a fluorinated PEG hydrogel studied by $1 \mathrm{H}$ molecular diffusion NMR and 19F spin diffusion NMR, Colloid and Polymer
Science. $288 \quad$ (2010) 1655-1663. https://doi.org/10.1007/s00396-010-2304-9.

[70] B. Wu, W. Chassé, K. Zick, M.D. Mantle, A. Heise, D.F. Brougham, V.M. Litvinov, The effect of hydrogen bonding on diffusion and permeability in UV-cured Polyacrylate-based networks for controlled release, Journal of Controlled Release. 327 (2020) 150-160. https://doi.org/10.1016/j.jconrel.2020.07.039.

[71] S. Lopes, M. Simeonova, P. Gameiro, M. Rangel, G. Ivanova, Interaction of 5Fluorouracil Loaded Nanoparticles with 1,2Dimyristoyl- sn -glycero-3-phosphocholine Liposomes Used as a Cellular Membrane Model, The Journal of Physical Chemistry B. 116 (2012) 667-675. https://doi.org/10.1021/jp210088n.

[72] Y. Kato, D. Artemov, Monitoring of release of cargo from nanocarriers by MRI/MR spectroscopy (MRS): Significance of $T_{2} / T_{2}$ * effect of iron particles, Magnetic Resonance in Medicine. 61 (2009) 1059-1065. https://doi.org/10.1002/mrm.21939.

[73] M. Carril, D. Padro, P. del Pino, C. CarrilloCarrion, M. Gallego, W.J. Parak, In situ detection of the protein corona in complex environments, Nature Communications. 8 (2017). https://doi.org/10.1038/s41467-01701826-4.

[74] Y.R. Perera, T.M. South, A.C. Hughes, A.N. Parkhurst, O.C. Williams, M.B. Davidson, C.A. Wilks, D.A. Mlsna, N.C. Fitzkee, Using NMR Spectroscopy to Measure Protein Binding Capacity on Gold Nanoparticles, Journal of Chemical Education. 97 (2020) 820-824.

https://doi.org/10.1021/acs.jchemed.9b00625.

[75] H. Kato, N. Shinohara, A. Nakamura, M. Horie, K. Fujita, K. Takahashi, H. Iwahashi, S. Endoh, S. Kinugasa, Characterization of fullerene colloidal suspension in a cell culture medium for in vitro toxicity assessment, Molecular BioSystems. 6 (2010) 1238. https://doi.org/10.1039/c002364g.

[76] S.C. Wilson, J.L. Baryza, A.J. Reynolds, K. Bowman, M.E. Keegan, S.M. Standley, N.P. Gardner, P. Parmar, V.O. Agir, S. Yadav, A. Zunic, C. Vargeese, C.C. Lee, S. Rajan, Real Time Measurement of PEG Shedding from Lipid Nanoparticles in Serum via NMR Spectroscopy, Molecular Pharmaceutics. 12 (2015) https://doi.org/10.1021/mp500400k.

[77] G. Lafitte, O. Söderman, K. Thuresson, J. Davies, PFG-NMR diffusometry: A tool for investigating the structure and dynamics of noncommercial purified pig gastric mucin in a wide range of concentrations, Biopolymers. 86 (2007) 165-175. https://doi.org/10.1002/bip.20717. 
[78] G. Lafitte, K. Thuresson, P. Jarwoll, M. Nydén, Transport Properties and Aggregation Phenomena of Polyoxyethylene Sorbitane Monooleate (Polysorbate 80) in Pig Gastrointestinal Mucin and Mucus, Langmuir. 23 (2007) 10933-10939. https://doi.org/10.1021/la701081s.

[79] M.A. Wisniewska, J.G. Seland, Investigating structure-dependent diffusion in hydrogels using spatially resolved NMR spectroscopy, Journal of Colloid and Interface Science. 533 (2019) 671-677. https://doi.org/10.1016/j.jcis.2018.08.112.

[80] X. Wang, Y. Chen, L. Xue, N. Pothayee, R. Zhang, J.S. Riffle, T.M. Reineke, L.A. Madsen, Diffusion of Drug Delivery Nanoparticles into Biogels Using TimeResolved MicroMRI, The Journal of Physical Chemistry Letters. 5 (2014) 3825-3830. https://doi.org/10.1021/jz501929u.

[81] I. Pereira de Sousa, B. Cattoz, M.D. Wilcox, P.C. Griffiths, R. Dalgliesh, S. Rogers, A. Bernkop-Schnürch, Nanoparticles decorated with proteolytic enzymes, a promising strategy to overcome the mucus barrier, European Journal of Pharmaceutics and Biopharmaceutics. 97 (2015) 257-264. https://doi.org/10.1016/j.ejpb.2015.01.008.

[82] P. Marshall, J.E.M. Snaar, Y.L. Ng, R.W. Bowtell, F.C. Hampson, P.W. Dettmar, E. Onsøyen, C.D. Melia, Localised mapping of water movement and hydration inside a developing bioadhesive bond, Journal of Controlled Release. 95 (2004) 435-446. https://doi.org/10.1016/j.jconrel.2003.12.007.

[83] S. Kwak, M. Lafleur, NMR Self-Diffusion of Molecular and Macromolecular Species in Dextran Solutions and Gels, Macromolecules. 36 (2003) 3189-3195. https://doi.org/10.1021/ma0213605.

[84] R.V. Galdino, C.A. Benevides, R.P. Tenório, Diffusion maps of Bacillus subtilis biofilms via magnetic resonance imaging highlight a complex network of channels, Colloids and Surfaces B: Biointerfaces. 190 (2020) 110905. https://doi.org/10.1016/j.colsurfb.2020.11090 5.

[85] M.P. Herrling, J. Weisbrodt, C.M. Kirkland, N.H. Williamson, S. Lackner, S.L. Codd, J.D. Seymour, G. Guthausen, H. Horn, NMR investigation of water diffusion in different biofilm structures, Biotechnology and Bioengineering. 114 (2017) 2857-2867. https://doi.org/10.1002/bit.26392.

[86] B. Ramanan, W.M. Holmes, W.T. Sloan, V.R. Phoenix, Application of Paramagnetically Tagged Molecules for Magnetic Resonance Imaging of Biofilm Mass Transport Processes, Applied and Environmental Microbiology. 76 (2010) 4027-4036. https://doi.org/10.1128/AEM.03016-09.
[87] P. Cao, E.X. Wu, In vivo diffusion MRS investigation of non-water molecules in biological tissues: Diffusion Spectroscopy in Biological Tissues, NMR in Biomedicine. 30 (2017) e3481. https://doi.org/10.1002/nbm.3481.

[88] P. Cao, S.-J. Fan, A.M. Wang, V.B. Xie, Z. Qiao, G.M. Brittenham, E.X. Wu, Diffusion magnetic resonance monitors intramyocellular lipid droplet size in vivo: Intramyocellular Lipid Diffusion, Magnetic Resonance in Medicine. 73 (2015) 59-69. https://doi.org/10.1002/mrm.25116.

[89] H. Lahrech, S. Zoula, R. Farion, C. Rémy, M. Décorps, In vivo measurement of the size of lipid droplets in an intracerebral glioma in the rat, Magnetic Resonance in Medicine. 45 (2001) 409-414. https://doi.org/10.1002/15222594(200103)45:3<409::AIDMRM1053>3.0.CO;2-O.

[90] M. Palombo, N. Shemesh, I. Ronen, J. Valette, Insights into brain microstructure from in vivo DW-MRS, NeuroImage. 182 (2018) 97-116. https://doi.org/10.1016/j.neuroimage.2017.11 .028 .

[91] J. Valette, C. Ligneul, C. Marchadour, C. Najac, M. Palombo, Brain Metabolite Diffusion from Ultra-Short to Ultra-Long Time Scales: What Do We Learn, Where Should We Go?, Frontiers in Neuroscience. 12 (2018). https://doi.org/10.3389/fnins.2018.00002.

[92] A.M. Wang, P. Cao, A. Yee, D. Chan, E.X. $\mathrm{Wu}$, Detection of extracellular matrix degradation in intervertebral disc degeneration by diffusion magnetic resonance spectroscopy: Detecting ECM Degradation by Diffusion MRS, Magnetic Resonance in Medicine. 73 (2015) 1703-1712. https://doi.org/10.1002/mrm.25289.

[93] E.A. Waters, J. Chen, X. Yang, H. Zhang, R. Neumann, A. Santeford, J. Arbeit, G.M. Lanza, S.A. Wickline, Detection of targeted perfluorocarbon nanoparticle binding using ${ }^{19}$ F diffusion weighted MR spectroscopy, Magnetic Resonance in Medicine. 60 (2008) 1232-1236. https://doi.org/10.1002/mrm.21794.

[94] M. de Smet, E. Heijman, S. Langereis, N.M. Hijnen, H. Grüll, Magnetic resonance imaging of high intensity focused ultrasound mediated drug delivery from temperature-sensitive liposomes: An in-vivo proof-of-concept study, Journal of Controlled Release. 150 (2011) 102-110. https://doi.org/10.1016/j.jconrel.2010.10.036.

[95] T. Tagami, W.D. Foltz, M.J. Ernsting, C.M. Lee, I.F. Tannock, J.P. May, S.-D. Li, MRI monitoring of intratumoral drug delivery and prediction of the therapeutic effect with a 
multifunctional thermosensitive liposome, Biomaterials. 32 (2011) 6570-6578. https://doi.org/10.1016/j.biomaterials.2011.0 5.029 .

[96] Z. Wang, X. Xue, Y. He, Z. Lu, B. Jia, H. Wu, Y. Yuan, Y. Huang, H. Wang, H. Lu, K.S. Lam, T.-Y. Lin, Y. Li, Novel RedoxResponsive Polymeric Magnetosomes with Tunable Magnetic Resonance Property for In Vivo Drug Release Visualization and DualModal Cancer Therapy, Advanced Functional $\begin{array}{llll}\text { Materials. } & 28 \quad \text { (2018) } & 1802159 .\end{array}$ https://doi.org/10.1002/adfm.201802159.

[97] Y. Onuki, I. Jacobs, D. Artemov, Y. Kato, Noninvasive visualization of in vivo release and intratumoral distribution of surrogate MR contrast agent using the dual MR contrast technique, Biomaterials. 31 (2010) 71327138.

https://doi.org/10.1016/j.biomaterials.2010.0 6.008 .
[98] Y. Jia, K. Geng, Y. Cheng, Y. Li, Y. Chen, R. Wu, Nanomedicine Particles Associated With Chemical Exchange Saturation Transfer Contrast Agents in Biomedical Applications, Frontiers in Chemistry. 8 (2020). https://doi.org/10.3389/fchem.2020.00326.

[99] R. Marasini, T.D. Thanh Nguyen, S. Aryal, Integration of gadolinium in nanostructure for contrast enhanced-magnetic resonance imaging, WIREs Nanomedicine and Nanobiotechnology. $12 \quad$ (2020). https://doi.org/10.1002/wnan.1580.

[100] R.G.D. Andrade, S.R.S. Veloso, E.M.S. Castanheira, Shape Anisotropic Iron OxideBased Magnetic Nanoparticles: Synthesis and Biomedical Applications, International Journal of Molecular Sciences. 21 (2020) 2455. https://doi.org/10.3390/ijms21072455. 